\title{
ABSENCE OF EIGENVALUES FOR THE GENERALIZED TWO-DIMENSIONAL PERIODIC DIRAC OPERATOR
}

\author{
L. I. DANILOV
}

\begin{abstract}
A generalized two-dimensional periodic Dirac operator is considered, with $L^{\infty}$-matrix-valued coefficients of the first-order derivatives and with complex matrix-valued potential. It is proved that if the matrix-valued potential has zero bound relative to the free Dirac operator, then the spectrum of the operator in question contains no eigenvalues.
\end{abstract}

\section{$\S 0$. INTRODUCTION}

Let $\mathcal{M}_{2}$ be the space of complex $(2 \times 2)$-matrices, $\widehat{I} \in \mathcal{M}_{2}$ the unit matrix, and

$$
\widehat{\sigma}_{1}=\left(\begin{array}{ll}
0 & 1 \\
1 & 0
\end{array}\right), \quad \widehat{\sigma}_{2}=\left(\begin{array}{cc}
0 & -i \\
i & 0
\end{array}\right), \quad \widehat{\sigma}_{3}=\left(\begin{array}{cc}
1 & 0 \\
0 & -1
\end{array}\right)
$$

the Pauli matrices. Consider the generalized Dirac operator

$$
\widehat{\mathcal{D}}=\sum_{j=1}^{2}\left(h_{j 1} \widehat{\sigma}_{1}+h_{j 2} \widehat{\sigma}_{2}\right)\left(-i \frac{\partial}{\partial x_{j}}\right),
$$

acting in $L^{2}\left(\mathbb{R}^{2} ; \mathbb{C}^{2}\right)$, with the domain $D(\widehat{\mathcal{D}})=H^{1}\left(\mathbb{R}^{2} ; \mathbb{C}^{2}\right)$. The functions $h_{j l} \in$ $L^{\infty}\left(\mathbb{R}^{2} ; \mathbb{R}\right)$ are assumed to be periodic with a (common) period lattice $\Lambda \subset \mathbb{R}^{2}$, and $0<\varepsilon \leq h_{11}(x) h_{22}(x)-h_{12}(x) h_{21}(x)$ for a.e. $x \in \mathbb{R}^{2}$. We denote by $\mathbb{L}_{\Lambda}\left(\mathbb{R}^{2}\right)$ the set of all $\Lambda$-periodic functions $W \in L_{\text {loc }}^{2}\left(\mathbb{R}^{2} ; \mathbb{C}\right)$ such that $W \varphi \in L^{2}\left(\mathbb{R}^{2}\right)$ for any $\varphi \in H^{1}\left(\mathbb{R}^{2}\right)$, and for every $\varepsilon>0$ there exists a number $C_{\varepsilon}(W) \geq 0$ satisfying

$$
\|W \varphi\|_{L^{2}\left(\mathbb{R}^{2}\right)} \leq \varepsilon\|\nabla \varphi\|_{L^{2}\left(\mathbb{R}^{2} ; \mathbb{C}^{2}\right)}+C_{\varepsilon}(W)\|\varphi\|_{L^{2}\left(\mathbb{R}^{2}\right)}
$$

for all $\varphi \in H^{1}\left(\mathbb{R}^{2}\right)$. If $V^{(l)} \in \mathbb{L}_{\Lambda}\left(\mathbb{R}^{2}\right), l=0,1,2,3$, then

$$
\widehat{\mathcal{D}}+\widehat{V}=\widehat{\mathcal{D}}+V^{(0)} \widehat{I}+\sum_{l=1}^{3} V^{(l)} \widehat{\sigma}_{l}
$$

is a closed operator in $L^{2}\left(\mathbb{R}^{2} ; \mathbb{C}^{2}\right)$ with domain $D(\widehat{\mathcal{D}}+\widehat{V})=D(\widehat{\mathcal{D}})=H^{1}\left(\mathbb{R}^{2} ; \mathbb{C}^{2}\right)$ (a $\Lambda$-periodic matrix-valued potential $\widehat{V}$ has zero bound relative to $\widehat{\mathcal{D}}$ if and only if $V^{(l)} \in$ $\left.\mathbb{L}_{\Lambda}\left(\mathbb{R}^{2}\right), l=0,1,2,3\right)$.

The following theorem is the main result of this paper.

Theorem 0.1. Let $h_{j l} \in L^{\infty}\left(\mathbb{R}^{2} ; \mathbb{R}\right), j, l=1,2$, be periodic functions with period lattice $\Lambda \subset \mathbb{R}^{2}$. Suppose that there exists $\varepsilon>0$ such that $\varepsilon \leq h_{11}(x) h_{22}(x)-h_{12}(x) h_{21}(x)$ for a.e. $x \in \mathbb{R}^{2}$. If $V^{(l)} \in \mathbb{L}_{\Lambda}\left(\mathbb{R}^{2}\right), l=0,1,2,3$, then the operator (0.1) has no eigenvalues.

2000 Mathematics Subject Classification. Primary 35P05.

Key words and phrases. Generalized periodic Dirac operator, matrix-valued potential, absolutely continuous spectrum. 
If, under the conditions of Theorem 0.1, the operator (0.1) is selfadjoint, then its spectrum is absolutely continuous. For the selfadjoint periodic elliptic differential operator, the absolute continuity of the spectrum is a consequence of the absence of eigenvalues (of infinite multiplicity); see [1] and also [2]. This fact is of general nature and is also valid for the generalized Dirac operator $\widehat{\mathcal{D}}+\widehat{V}$.

The first results about the absence of eigenvalues in the spectrum of the periodic Dirac operator were obtained in [3]- 5 . For $n \geq 2$, consider the $n$-dimensional periodic (with period lattice $\Lambda \subset \mathbb{R}^{n}$ ) Dirac operator

$$
\sum_{j=1}^{n} \widehat{\alpha}_{j}\left(-i \frac{\partial}{\partial x_{j}}-A_{j}\right)+\widehat{V}+\widehat{V}_{0}, \quad x \in \mathbb{R}^{n}
$$

where

$$
\widehat{V}=V \widehat{I}, \quad \widehat{V}_{0}=m \widehat{\alpha}_{n+1}, \quad m \in \mathbb{R},
$$

the $\widehat{\alpha}_{j}, j=1, \ldots, n+1$, are Hermitian $(M \times M)$-matrices satisfying the anticommutation relations $\widehat{\alpha}_{j} \widehat{\alpha}_{l}+\widehat{\alpha}_{l} \widehat{\alpha}_{j}=2 \delta_{j l} \widehat{I}$ ( $\delta_{j l}$ is the Kronecker symbol), and $\widehat{I}$ is the unit $(M \times M)$ matrix $(M \in 2 \mathbb{N})$. The components $A_{j}$ of the vector-valued (magnetic) potential and the scalar (electric) potential $V$ are real-valued periodic functions with period lattice $\Lambda \subset \mathbb{R}^{n}$; let $K$ be the standard fundamental domain of $\Lambda$. In [5, 6], the absolute continuity of the spectrum of the operator $(0.2),(0.3)$ was proved for all $n \geq 2$ under the conditions $V \in C\left(\mathbb{R}^{n}\right), A \in L^{\infty}\left(\mathbb{R}^{n} ; \mathbb{R}^{n}\right)$, and

$$
\||A|\|_{L^{\infty}\left(\mathbb{R}^{n}\right)}<\max _{\gamma \in \Lambda \backslash\{0\}} \frac{\pi}{|\gamma|}
$$

$\left(|x|\right.$ is the length of the vector $\left.x \in \mathbb{R}^{n}\right)$. In subsequent papers, the restriction on the periodic scalar potential $V$ has been relaxed. The spectrum of the operator $(0.2),(0.3)$ is absolutely continuous if (at least) one of the following conditions is satisfied:

1) $n=2, V \in L^{q}(K), q>2$, and the vector-valued potential $A \in L^{\infty}\left(\mathbb{R}^{2} ; \mathbb{R}^{2}\right)$ satisfies (0.4) (see [7]);

2) $n \geq 3, A \equiv 0$, and $\sum_{N}\left|V_{N}\right|^{p}<+\infty$, where the $V_{N}$ are the Fourier coefficients of $V, p \in\left[1, q_{n}\left(q_{n}-1\right)^{-1}\right)$, and the $q_{n}>n$ are some numbers, the smallest values of which were presented for $n \geq 4$ in $\left[8\right.$. (the numbers $q_{n}$ are found as the largest roots of the algebraic equations

$$
q^{4}-\left(3 n^{2}-4 n-1\right) q^{3}+2\left(4 n^{2}-6 n-3\right) q^{2}-\left(9 n^{2}-16 n-4\right) q-4 n(n-2)=0,
$$

$q_{3} \simeq 11.645, n^{-2} q_{n} \rightarrow 3$ as $n \rightarrow+\infty$; for the first time the above condition on the Fourier coefficients appeared in 4 for $n=3$ );

3) $n=3, V \in L^{q}(K), q>3$, and the vector-valued potential $A \in L^{\infty}\left(\mathbb{R}^{3} ; \mathbb{R}^{3}\right)$ satisfies (0.4) (see 9 );

4) $n \geq 2, V \in L^{2}(K), A \in L^{\infty}\left(\mathbb{R}^{n} ; \mathbb{R}^{n}\right)$, there exists a vector $\gamma \in \Lambda \backslash\{0\}$ such that $\||A|\|_{L^{\infty}\left(\mathbb{R}^{n}\right)}<\pi|\gamma|^{-1}$, and the map

$$
\mathbb{R}^{n} \ni x \rightarrow\{[0,1] \ni t \rightarrow V(x+t \gamma)\} \in L^{2}([0,1])
$$

is continuous; see [10]. (In particular, under an appropriate choice of $\gamma \in \Lambda \backslash\{0\}$, the latter condition is satisfied if $V$ is an arbitrary piecewise continuous scalar potential with piecewise analytic discontinuity surfaces; such potentials were considered in [3.)

Some other conditions on the scalar potential $V$ and a small vector-valued potential $A$ can be found in [10].

The periodic Dirac operator with a nonsmall vector-valued potential $A$ was studied in [11 - 13]. In [12, the absolute continuity of the spectrum of the operator $(0.2)$ was proved under the conditions $\widehat{V}=V \widehat{I}, \widehat{V}_{0}=V_{0} \widehat{\alpha}_{n+1}$, where $V, V_{0} \in L^{q}(K, \mathbb{R}), A \in L^{q}\left(K ; \mathbb{R}^{2}\right)$ 
$(q>2)$ for $n=2$, and under the conditions $V, V_{0} \in C\left(\mathbb{R}^{n} ; \mathbb{R}\right), A \in C^{2 n+3}\left(\mathbb{R}^{n} ; \mathbb{R}^{n}\right)$ for $n \geq 3$. For $n=2$, the proof is based on the results of the papers [14, 15, where the periodic Schrödinger operator

$$
\sum_{j=1}^{2}\left(-i \frac{\partial}{\partial x_{j}}-A_{j}\right)^{2}+V, \quad x \in \mathbb{R}^{2},
$$

was treated. For the operator (0.5), the absolute continuity of the spectrum was proved first for $V \in L_{\text {loc }}^{2}\left(\mathbb{R}^{2} ; \mathbb{R}\right), A \in C\left(\mathbb{R}^{2} ; \mathbb{R}^{2}\right)$ in [14, and then in [15] for the more general case where $V \in L_{\text {loc }}^{q}\left(\mathbb{R}^{2} ; \mathbb{R}\right), A \in L_{\text {loc }}^{2 q}\left(\mathbb{R}^{2} ; \mathbb{R}^{2}\right), q>1$. For the periodic operator (0.2) with $n=2$, a similar result (as in 12]) was obtained in 11] (it was assumed, however, that $V_{0} \equiv m=$ const, but the proof carries over to functions $V_{0} \in L^{q}(K), q>2$, without essential modifications). The methods used in [11] were the same as in [7. More general conditions on $V, V_{0}$, and $A$ (for $n=2$ ) were obtained in [16): it suffices to require that the functions $V^{2} \ln (1+|V|), V_{0}^{2} \ln \left(1+\left|V_{0}\right|\right)$, and $|A|^{2} \ln ^{q}(1+|A|)$ belong to $L^{1}(K)$ for some $q>1$. For $n \geq 3$, the results of [12] were based on Sobolev's paper [17, where the absolute continuity of the spectrum was proved for the Schrödinger operator with a periodic vector-valued potential $A \in C^{2 n+3}\left(\mathbb{R}^{n} ; \mathbb{R}^{n}\right)$. The latter condition was relaxed by Kuchment and Levendorskiu in [2] (and also by Sobolev; see the remark at the end of the survey [18]): it suffices to require that $A \in H_{\mathrm{loc}}^{q}\left(\mathbb{R}^{n} ; \mathbb{R}^{n}\right), 2 q>3 n-2$, which makes it possible to relax accordingly the constraint on the vector-valued potential $A$ also for the periodic Dirac operator (see [12, 18]).

Let $\mathfrak{M}_{h}, h>0$, be the set of all even Borel signed measures (charges) $\mu$ on $\mathbb{R}$ (with finite full variation) for which $\int_{\mathbb{R}} e^{i p t} d \mu(t)=1$ for every $p \in(-h, h)$. In [13], it was proved that the spectrum of the periodic (with period lattice $\Lambda$ ) Dirac operator (0.2) is absolutely continuous for $n \geq 3$ if the following conditions are fulfilled:

1) the $(M \times M)$-matrix-valued functions $\widehat{V}$ and $\widehat{V}_{0}$ are Hermitian and continuous, and $\widehat{V}(x) \widehat{\alpha}_{j}=\widehat{\alpha}_{j} \widehat{V}(x), \widehat{V}_{0}(x) \widehat{\alpha}_{j}=-\widehat{\alpha}_{j} \widehat{V}_{0}(x)$ for all $x \in \mathbb{R}^{n}, j=1, \ldots, n$;

2) $A \in C\left(\mathbb{R}^{n} ; \mathbb{R}^{n}\right)$ and there exists a vector $\gamma \in \Lambda \backslash\{0\}$ and a measure $\mu \in \mathfrak{M}_{h}, h>0$, such that for every $x \in \mathbb{R}^{n}$ and every unit vector $e \in \mathbb{R}^{n}$ with $(e, \gamma)=0$ we have

$$
\left|\int_{\mathbb{R}} d \mu(t) \int_{0}^{1} A(x-\xi \gamma-t e) d \xi-A_{0}\right|<\frac{\pi}{|\gamma|},
$$

where $A_{0}=v^{-1}(K) \int_{K} A(x) d^{n} x, v(K)$ is the volume of the fundamental domain $K$, and $(.,$.$) is the scalar product in \mathbb{R}^{n}$.

For a periodic vector-valued potential $A \in C\left(\mathbb{R}^{n} ; \mathbb{R}^{n}\right)$, condition (0.6) is fulfilled (under an appropriate choice of $\gamma \in \Lambda \backslash\{0\}$ and measure $\mu \in \mathfrak{M}_{h}, h>0$ ) whenever $A \in$ $H_{\text {loc }}^{q}\left(\mathbb{R}^{n} ; \mathbb{R}^{n}\right), 2 q>n-2$, and also in the case where $\sum_{N}\left|A_{N}\right|_{\mathbb{C}^{n}}<+\infty$, where the $A_{N}$ are the Fourier coefficients of $A$ (see [13, 19]). The results of [13] were used in [20] in order to prove the absolute continuity of the spectrum of the periodic Schrödinger operator

$$
\sum_{j=1}^{n}\left(-i \frac{\partial}{\partial x_{j}}-A_{j}\right)^{2}+V, \quad x \in \mathbb{R}^{n}, \quad n \geq 3
$$

with a vector-valued potential $A \in C^{1}\left(\mathbb{R}^{n} ; \mathbb{R}^{n}\right)$ satisfying condition 2$)$ and with the scalar potential $V \in L_{w}^{p}(K ; \mathbb{R})$ for which $t(\operatorname{meas}\{x \in K:|V(x)|>t\})^{1 / p} \rightarrow 0$ as $t \rightarrow+\infty$, where $p=n / 2$ for $3 \leq n \leq 6$ and $p=n-3$ for $n \geq 7$, meas standing for Lebesgue measure). The multidimensional periodic Schrödinger operator was studied in many papers; the relevant facts and references can be found in [2, 18] and 21]-25].

Let $\mathbb{G}$ denote the set of all continuously differentiable and monotone nondecreasing functions $g:(0,+\infty) \rightarrow(0,+\infty)$ such that $\int_{0}^{1}(r g(r))^{-1} d r<+\infty$ and $g(r / 2) / g(r) \rightarrow 1$ 
as $r \rightarrow+0$. We write $L^{2}\{g, \Lambda\}, g \in \mathbb{G}$, for the Banach space of periodic (with period lattice $\left.\Lambda \subset \mathbb{R}^{2}\right)$ functions $W \in L_{\text {loc }}^{2}\left(\mathbb{R}^{2} ; \mathbb{C}\right)$ such that

$$
\|W\|_{L^{2}\{g, \Lambda\}}^{2}=\sup _{x \in \mathbb{R}^{2}} \int_{y:|x-y| \leq 1} g(|x-y|)|W(y)|^{2} d^{2} y<+\infty .
$$

If $g \in \mathbb{G}$, then $g(r)(\ln r)^{-1} \rightarrow-\infty$ as $r \rightarrow+0$; therefore, for any $W \in L^{2}\{g, \Lambda\}$ the function $W^{2}$ belongs to the Kato class $K_{2}$ (see [26), which implies that $W \in \mathbb{L}_{\Lambda}\left(\mathbb{R}^{2}\right)$.

For $n=2$, the operator $\widehat{\mathcal{D}}+\widehat{V}$ (see $(0.1)$ ) was considered in 27 in the case where $h_{j l} \in C^{\infty}\left(\mathbb{R}^{2}\right), j, l=1,2, V^{(l)} \in C^{\infty}\left(\mathbb{R}^{2} ; \mathbb{R}\right)$ for $l=1,2$, and $V^{(l)}, \partial V^{(l)} / \partial x_{j} \in L^{\infty}\left(\mathbb{R}^{2}\right)$ for $l=0,3$ and $j=1,2$. In [28, a special case of Theorem 0.1 was proved: under the same conditions on $h_{j l}$, it was assumed that $\widehat{V} \in L_{\mathrm{loc}}^{q}\left(\mathbb{R}^{2} ; \mathcal{M}_{2}\right), q>2$. The latter result was improved in 29] (and was announced in [30]): it suffices to require that $V^{(0)}, V^{(3)} \in \mathbb{L}_{\Lambda}\left(\mathbb{R}^{2}\right)$ and $V^{(1)}, V^{(2)} \in L^{2}\{g, \Lambda\} \subset \mathbb{L}_{\Lambda}\left(\mathbb{R}^{2}\right)$ for some $g \in \mathbb{G}$.

The methods employed in the proof of Theorem 0.1 can also be used for the proof of the absolute continuity of the spectrum of the two-dimensional periodic Schrödinger operator

$$
\sum_{j, l=1}^{2}\left(-i \frac{\partial}{\partial x_{j}}-A_{j}\right) G_{j l}\left(-i \frac{\partial}{\partial x_{l}}-A_{l}\right)+V, \quad x \in \mathbb{R}^{2},
$$

with variable metric; here $G=\left(G_{j l}\right)_{j, l=1,2}$ is a real symmetric positive definite matrixvalued function (a metric) with $G, G^{-1} \in L^{\infty}\left(\mathbb{R}^{2} ; \mathcal{M}_{2}\right)$. The functions $A, V$, and $G$ are periodic with a common period lattice $\Lambda \subset \mathbb{R}^{2}$. For the first time, the absolute continuity of the spectrum of the operator (0.7) was proved by Morame in 27] under the conditions $A \in C^{\infty}\left(\mathbb{R}^{2} ; \mathbb{R}^{2}\right), V \in L^{\infty}\left(\mathbb{R}^{2} ; \mathbb{R}\right)$, and $G \in C^{\infty}\left(\mathbb{R}^{2} ; \mathcal{M}_{2}\right)$, det $G \equiv 1$. Later on, in the case where $G \in C^{m+\alpha}\left(\mathbb{R}^{2} ; \mathcal{M}_{2}\right), m \in \mathbb{Z}_{+}=\mathbb{N} \cup\{0\}, \alpha \in(0,1)$, Kuchment and Levendorskiu [2] proved the existence of periodic isothermal coordinates $y(x) \in C^{m+1+\alpha}\left(\mathbb{R}^{2} ; \mathbb{R}^{2}\right)$ that reduce the matrix-valued function $G$ to a scalar form; the use of such coordinates allowed them to relax the restrictions on $A, V$, and $G$ by reducing the problem to the case of a constant matrix $G$. The periodic isothermal coordinates were applied in a series of papers by Birman, Suslina, and Shterenberg. In 33, the absolute continuity of the spectrum of the operator $(0.7)$ was proved for $G \in W_{2 q, \text { loc }}^{2}\left(\mathbb{R}^{2} ; \mathcal{M}_{2}\right), A \in L^{2 q}\left(K ; \mathbb{R}^{2}\right), q>1$, and $V=V_{1}+\sigma \delta_{\Sigma}$, where $V_{1} \in L^{q}(K ; \mathbb{R}), \Sigma$ is a periodic system of piecewise smooth curves, $\delta_{\Sigma}$ is the $\delta$-function concentrated on $\Sigma$, and $\sigma \in L_{\mathrm{loc}}^{q}(\Sigma ; \mathbb{R})$. In the subsequent papers [34-37, Shterenberg relaxed the conditions imposed on $A, V$, and $G$. The following conditions were given in [36]:

$$
\begin{gathered}
\operatorname{det} G \in H_{\mathrm{loc}}^{1}\left(\mathbb{R}^{2}\right), \quad \frac{\partial}{\partial x_{j}} \operatorname{det} G \in \mathbb{L}_{\Lambda}\left(\mathbb{R}^{2}\right), \quad j=1,2, \\
|A|^{2} \widetilde{g}(|A|) \in L_{\mathrm{loc}}^{1}\left(\mathbb{R}^{2}\right),
\end{gathered}
$$

where $\tilde{g}(t)=l(t) \doteq l_{m}^{q}(t) \prod_{i=1}^{m-1} l_{i}(t), m \in \mathbb{N}, q>1, l_{1}(t)=1+\ln (1+t), l_{i}(t)=$ $1+\ln l_{i-1}(t), i=2, \ldots, m, t \geq 0$, and the scalar potential $V$ is defined as the distribution $d \mu / d^{2} x$, where $\mu$ is a periodic Borel signed measure satisfying some additional conditions (which occur also in 35 ); in this situation the closure of the quadratic form $\mathcal{V}(\varphi, \varphi)=\int_{\mathbb{R}^{2}}|\varphi|^{2} d \mu, \varphi \in C_{0}^{\infty}\left(\mathbb{R}^{2}\right)$, may fail to be bounded relative to the form $\|\nabla \varphi\|_{L^{2}\left(\mathbb{R}^{2} ; \mathbb{C}^{2}\right)}^{2}, \varphi \in H^{1}\left(\mathbb{R}^{2}\right) \subset L^{2}\left(\mathbb{R}^{2}\right)$. Finally, in 37], the restriction (0.9) on the vectorvalued potential $A$ was relaxed to $A_{j} \in \mathbb{L}_{\Lambda}\left(\mathbb{R}^{2}\right), j=1,2$. It should be mentioned that in [36, 37. Shterenberg studied also a generalization of the operator (0.7) obtained by the incorporation of weight functions. The results pertaining to the absolute continuity of the 
spectrum of the periodic Schrödinger operator were applied to the study of the spectrum of the Schrödinger operator in periodic waveguides (see [38, 39] and also [40, 41]).

In [30] (without using periodic isothermal coordinates, and with the help of results on the generalized two-dimensional periodic Dirac operator), it was proved that no eigenvalues are present in the spectrum of the periodic operator (0.7) if the following conditions are fulfilled: $G$ satisfies (0.8), $A_{j} \in L^{2}\{g, \Lambda\}, j=1,2$, for some $g \in \mathbb{G}$, and the scalar potential $V$ is defined via a quadratic form $\mathcal{V}(\varphi, \varphi), \varphi \in H^{1}\left(\mathbb{R}^{2}\right)$; this form has zero bound relative to the form $\|\nabla \varphi\|_{L^{2}\left(\mathbb{R}^{2} ; \mathbb{C}^{2}\right)}^{2}, \varphi \in H^{1}\left(\mathbb{R}^{2}\right) \subset L^{2}\left(\mathbb{R}^{2}\right)$, and is such that $\mathcal{V}(\psi(.-\gamma), \varphi(.-\gamma))=\mathcal{V}(\psi, \varphi)$ for all $\psi, \varphi \in H^{1}\left(\mathbb{R}^{2}\right), \gamma \in \Lambda$, and $\mathcal{V}\left(e^{i(k, x)} \psi, e^{i(k, x)} \varphi\right)=$ $\mathcal{V}(\psi, \varphi)$ for all $\psi, \varphi \in H^{1}\left(\mathbb{R}^{2}\right)$ and $k \in \mathbb{R}^{2}$ (if the form $\mathcal{V}(\varphi, \varphi)$ is Hermitian, then it may fail to coincide with $\int_{\mathbb{R}^{2}}|\varphi|^{2} d \mu$ for $\varphi \in C_{0}^{\infty}\left(\mathbb{R}^{2}\right)$, where $\mu$ is a periodic (locally finite) Borel signed measure [29]). In [30, the functions $A_{j}, j=1,2$, can be complex-valued, and the form $\mathcal{V}$ is not assumed to be Hermitian.

If the function $[0,+\infty) \ni t \rightarrow \widetilde{g}(t) \in[0,+\infty)$ is monotone nondecreasing and the function $(0,+\infty) \ni t \rightarrow \widetilde{g}\left(t^{-1}\right)$ belongs to $\mathbb{G}$ (in particular, this is true if $\widetilde{g}()=.l($.$) ),$ then for every periodic vector-valued potential $A \in L_{\text {loc }}^{2}\left(\mathbb{R}^{2} ; \mathbb{C}^{2}\right)$ satisfying $(0.9)$ there exists $g \in \mathbb{G}$ such that $A_{j} \in L^{2}\{g, \Lambda\}, j=1,2$ (see [31]). Therefore, the condition imposed in [30] on the vector-valued potential $A$ is less restrictive than (0.9) (with $\widetilde{g}$ as indicated). In [32] (as well as in [37]), the constraint on $A$ was relaxed up to $A_{j} \in \mathbb{L}_{\Lambda}\left(\mathbb{R}^{2}\right)$, $j=1,2$.

\section{$\S 1$. Notation And the Main Statements}

Since the change $V^{(0)}-\lambda \rightarrow V^{(0)}$ reduces the operator $\widehat{\mathcal{D}}+\widehat{V}-\lambda \widehat{I}$, where $\widehat{I}$ is the identity operator on $L^{2}\left(\mathbb{R}^{2} ; \mathbb{C}^{2}\right)$ and $\lambda \in \mathbb{C}$, to the operator $\widehat{\mathcal{D}}+\widehat{V}$, it follows that in the proof of Theorem 0.1 it suffices to check the absence of the eigenvalue 0 . Also, we may assume that $\Lambda=\mathbb{Z}^{2}$ (an appropriate linear change of variables can be made, preserving the form of the operator $\widehat{\mathcal{D}}+\widehat{V})$. Denote $\mathbb{L}\left(\mathbb{R}^{2}\right) \doteq \mathbb{L}_{\mathbb{Z}^{2}}\left(\mathbb{R}^{2}\right), K=[0,1)^{2}$. Let $0<q \leq$ $p<+\infty$, let $F \geq 0$, and let $\Gamma(p, q, F)$ denote the set of ordered collections $\{\mathcal{F}, \mathcal{G}, \mathcal{H}\}$ of $\mathbb{Z}^{2}$-periodic functions $\mathcal{F}, \mathcal{G}, \mathcal{H}$ in $L^{\infty}\left(\mathbb{R}^{2} ; \mathbb{R}\right)$ such that $q \leq \mathcal{G}(x) \leq p, q \leq \mathcal{H}(x) \leq p$, and $|\mathcal{F}(x)| \leq F$ for a.e. $x \in \mathbb{R}^{2}$. We put $\Gamma=\bigcup_{p, q, F} \Gamma(p, q, F)$. Multiplying the generalized Dirac operator (0.1) from the left by the (unitary) matrix-valued function

$$
\left(h_{21}^{2}(x)+h_{22}^{2}(x)\right)^{-1 / 2}\left(h_{22}(x) \widehat{I}-i h_{21}(x) \widehat{\sigma}_{3}\right), \quad x \in \mathbb{R}^{2},
$$

we obtain the operator

$$
\widehat{\mathcal{D}}+\widehat{V}=\left(\mathcal{G} \widehat{\sigma}_{1}+\mathcal{F} \widehat{\sigma}_{2}\right)\left(-i \frac{\partial}{\partial x_{1}}\right)+\mathcal{H} \widehat{\sigma}_{2}\left(-i \frac{\partial}{\partial x_{2}}\right)+\widehat{V}
$$

for which $\{\mathcal{F}, \mathcal{G}, \mathcal{H}\} \in \Gamma$ and the (new) periodic matrix-valued potential $\widehat{V}$ satisfies the assumptions of Theorem 0.1. Therefore, Theorem 0.1 is a direct consequence of the next Theorem 1.1.

Theorem 1.1. Suppose $\{\mathcal{F}, \mathcal{G}, \mathcal{H}\} \in \Gamma$ and

$$
\widehat{V}=V^{(0)} \widehat{I}+\sum_{l=1}^{3} V^{(l)} \widehat{\sigma}_{l}
$$

with $V^{(l)} \in \mathbb{L}\left(\mathbb{R}^{2}\right), l=0,1,2,3$. Then the generalized Dirac operator (1.1), acting in $L^{2}\left(\mathbb{R}^{2} ; \mathbb{C}^{2}\right)$ and defined on the Sobolev class $H^{1}\left(\mathbb{R}^{2} ; \mathbb{C}^{2}\right)$, is invertible (i.e., $\lambda=0$ is not an eigenvalue of this operator).

For the proof of Theorem 1.1, we apply Thomas' method (originating from 42 and used for checking the absence of eigenvalues in the spectrum of periodic elliptic differential 
operators). With the help of this method, in this section we reduce Theorem 1.1 to Theorem 1.2.

As a preliminary, we introduce some notation and several definitions. The Fourier coefficients of a function $\varphi \in L^{1}\left(K ; \mathbb{C}^{d}\right), d=1,2$, will be denoted by

$$
\varphi_{N}=\int_{K} \varphi(x) e^{-2 \pi i(N, x)} d^{2} x, \quad N \in \mathbb{Z}^{2} .
$$

Let $\widetilde{C}(K), \widetilde{C}^{1}(K)$, and $\widetilde{H}^{1}(K)$ be the spaces of functions $\varphi: K \rightarrow \mathbb{C}$, the $\mathbb{Z}^{2}$-periodic extensions of which belong to $C\left(\mathbb{R}^{2}\right), C^{1}\left(\mathbb{R}^{2}\right)$, and the Sobolev class $H_{\text {loc }}^{1}\left(\mathbb{R}^{2}\right)$, respectively; by $\widetilde{C}_{0}(K), \widetilde{C}_{0}^{1}(K)$, and $\widetilde{H}_{0}^{1}(K)$ we denote the corresponding subspaces of functions $\varphi$ such that $\varphi_{0}=\int_{K} \varphi(x) d^{2} x=0 ; \widetilde{H}^{1}\left(K ; \mathbb{C}^{2}\right)=\left(\widetilde{H}^{1}(K)\right)^{2}$. In what follows, we identify functions defined on $K$ with their $\mathbb{Z}^{2}$-periodic extensions to $\mathbb{R}^{2}$. The norms and scalar products in $\mathbb{C}^{d}, L^{2}\left(\mathbb{R}^{2} ; \mathbb{C}^{d}\right)$, and $L^{2}\left(K ; \mathbb{C}^{d}\right), d=1,2$, are standard (as a rule, we do not indicate a particular space in the notation for its norm and scalar product). The scalar products are assumed to be linear in the second argument; $\nabla=\left(\partial / \partial x_{1}, \partial / \partial x_{2}\right)$, and meas is the Lebesgue measure.

Let $\{\mathcal{F}, \mathcal{G}, \mathcal{H}\} \in \Gamma(p, q, F)$. For all $k=\left(k_{1}, k_{2}\right) \in \mathbb{R}^{2}$ and all $\varkappa=\left(\varkappa_{1}, \varkappa_{2}\right) \in \mathbb{R}^{2}$, we introduce the operators

$$
\widehat{\mathcal{D}}(k+i \varkappa)=\left(\mathcal{G} \widehat{\sigma}_{1}+\mathcal{F} \widehat{\sigma}_{2}\right)\left(k_{1}+i \varkappa_{1}-i \frac{\partial}{\partial x_{1}}\right)+\mathcal{H} \widehat{\sigma}_{2}\left(k_{2}+i \varkappa_{2}-i \frac{\partial}{\partial x_{2}}\right),
$$

acting in $L^{2}\left(K ; \mathbb{C}^{2}\right)$, with $D(\widehat{\mathcal{D}}(k+i \varkappa))=\widetilde{H}^{1}\left(K ; \mathbb{C}^{2}\right)$. Put

$$
\widehat{d}_{ \pm}(k+i \varkappa)=(\mathcal{G} \pm i \mathcal{F})\left(k_{1}+i \varkappa_{1}-i \frac{\partial}{\partial x_{1}}\right) \pm i \mathcal{H}\left(k_{2}+i \varkappa_{2}-i \frac{\partial}{\partial x_{2}}\right),
$$

$D\left(\widehat{d}_{ \pm}(k+i \varkappa)\right)=\widetilde{H}^{1}(K) \subset L^{2}(K)$;

$$
\widehat{\mathcal{D}}(k+i \varkappa)=\left(\begin{array}{cc}
0 & \widehat{d}_{-}(k+i \varkappa) \\
\widehat{d}_{+}(k+i \varkappa) & 0
\end{array}\right) .
$$

There exist numbers $c_{1}=c_{1}(p, q, F)>0$ and $c_{2}=c_{2}(p, q, F) \geq c_{1}$ such that for all $k \in \mathbb{R}^{2}$ and all $\varphi \in \widetilde{H}^{1}(K)$ we have

$$
c_{1} \sum_{j=1}^{2}\left\|\left(k_{j}-i \frac{\partial}{\partial x_{j}}\right) \varphi\right\|^{2} \leq\left\|\widehat{d}_{ \pm}(k) \varphi\right\|^{2} \leq c_{2} \sum_{j=1}^{2}\left\|\left(k_{j}-i \frac{\partial}{\partial x_{j}}\right) \varphi\right\|^{2}
$$

(see, e.g., [29]).

Relations (1.2) and (1.3) imply that the operators $\widehat{\mathcal{D}}(k), k \in \mathbb{R}^{2}$, are closed, and if $k \notin 2 \pi \mathbb{Z}^{2}$, then their range $R(\widehat{\mathcal{D}}(k))$ coincides with the space $L^{2}\left(K ; \mathbb{C}^{2}\right)$, ker $\widehat{\mathcal{D}}(k)=\{0\}$, and the inverse operators $\widehat{\mathcal{D}}^{-1}(k)$ are compact (see (1.2), (1.3), and the properties of $\widehat{d}_{ \pm}(k)$ presented in $\left.\S 2\right)$.

The generalized Dirac operator $\widehat{\mathcal{D}}+\widehat{V}$ of the form (1.1) is unitarily equivalent to the direct integral

$$
\int_{2 \pi K}^{\oplus}(\widehat{\mathcal{D}}(k)+\widehat{V}) \frac{d^{2} k}{(2 \pi)^{2}}
$$

acting in

$$
\int_{2 \pi K}^{\oplus} L^{2}\left(K ; \mathbb{C}^{2}\right) \frac{d^{2} k}{(2 \pi)^{2}}
$$

(the vector $k=\left(k_{1}, k_{2}\right) \in 2 \pi K \subset \mathbb{R}^{2}$ is called the quasimomentum). The unitary equivalence mentioned above is established via the Gelfand transformation [43, 44] (for periodic Dirac operators, see also [9, 12]). The matrix-valued potential $\widehat{V}$, viewed as 
acting in $L^{2}\left(K ; \mathbb{C}^{2}\right)$, has zero bound relative to the operators $\widehat{\mathcal{D}}(k), k \in \mathbb{R}^{2}$; therefore, the operators $\widehat{V} \widehat{\mathcal{D}}^{-1}(k)$ are compact for all $k \in \mathbb{R}^{2} \backslash 2 \pi \mathbb{Z}^{2}$. Fix a vector $k^{0} \in \mathbb{R}^{2} \backslash 2 \pi \mathbb{Z}^{2}$. Since

$$
(\widehat{\mathcal{D}}(k)+\widehat{V}) \widehat{\mathcal{D}}^{-1}\left(k^{0}\right)=\widehat{I}+\widehat{S}(k), \quad k \in \mathbb{R}^{2},
$$

where $\widehat{I}$ is the identity operator in $L^{2}\left(K ; \mathbb{C}^{2}\right)$, and the operator

$$
\widehat{S}(k)=\left(\left(\mathcal{G} \widehat{\sigma}_{1}+\mathcal{F} \widehat{\sigma}_{2}\right)\left(k_{1}-k_{1}^{0}\right)+\mathcal{H} \widehat{\sigma}_{2}\left(k_{2}-k_{2}^{0}\right)+\widehat{V}\right) \widehat{\mathcal{D}}^{-1}\left(k^{0}\right)
$$

is compact, the representation of the operator (1.1) in the form of a direct integral (1.4) and the analytic Fredholm theorem imply that if $\lambda=0$ is an eigenvalue of $\widehat{\mathcal{D}}+\widehat{V}$, then $\lambda=0$ is an eigenvalue of each of the operators $\widehat{\mathcal{D}}(k+i \varkappa)+\widehat{V}$ (with the domain $\left.D(\widehat{\mathcal{D}}(k+i \varkappa)+\widehat{V})=\widetilde{H}^{1}\left(K ; \mathbb{C}^{2}\right) \subset L^{2}\left(K ; \mathbb{C}^{2}\right)\right)$ for all $k+i \varkappa \in \mathbb{C}^{2}$ (see [1] and 44, XIII.16]). Consequently, for the proof of Theorem 1.1 it suffices to find a complex vector $k+i \varkappa \in \mathbb{C}^{2}$ such that $\operatorname{ker}(\widehat{\mathcal{D}}(k+i \varkappa)+\widehat{V})=\{0\}$. Thus, Theorem 1.1 is implied by the following statement.

Theorem 1.2. Suppose $\{\mathcal{F}, \mathcal{G}, \mathcal{H}\} \in \Gamma(p, q, F)$,

$$
\widehat{V}(.)=V^{(0)}(.) \widehat{I}+\sum_{l=1}^{3} V^{(l)}(.) \widehat{\sigma}_{l},
$$

$V^{(l)} \in \mathbb{L}\left(\mathbb{R}^{2}\right), l=0,1,2,3$. Then there are vectors $k^{\prime}, \varkappa^{\prime} \in \mathbb{R}^{2}$, and a unit vector $e=\left(e_{1}, e_{2}\right) \in \mathbb{R}^{2}$ with $e_{1}>0$ such that for some numbers $\widetilde{\mu}>0$ as large as we wish the following is true: for all $k \in \mathbb{R}^{2}$ with $k_{1}=\pi$ and all vector-valued functions $\varphi \in$ $\widetilde{H}^{1}\left(K ; \mathbb{C}^{2}\right)$ we have

$$
\left\|\left(\widehat{\mathcal{D}}\left(k+k^{\prime}+i\left(\widetilde{\mu} e+\varkappa^{\prime}\right)\right)+\widehat{V}\right) \varphi\right\| \geq e^{-c \widetilde{\mu}}\|\varphi\|,
$$

where $c=c(p, q, F)>0$.

The proof of Theorem 1.2 is presented in $\S 5$. It is based on Theorems 3.1 and 5.1. proved in $\S 3$ and $\S 6$, respectively. In $\S 2$, for the operators $\widehat{d}_{ \pm}(k)$ we list the properties needed for what follows. In $\S 3$ we prove that the Dirac operator $\widehat{\mathcal{D}}(0)+\widehat{V}$ with a matrixvalued potential of a special form is similar to the Dirac operator $\widehat{\mathcal{D}}(k+i \varkappa)$ for some vectors $k, \varkappa \in \mathbb{R}^{2}$. In $\S 4$ we collect the auxiliary statements to be used either in the proof of Theorem 1.2, or (mainly) in the proof of Theorem 5.1. The estimates proved in Theorem 5.1 for the Dirac operator $\widehat{\mathcal{D}}(k)+\widehat{V}$ with a matrix-valued potential of a special form are used in the proof of Theorem 1.2. In $\S 6$, Theorem 5.1 is deduced from Theorem 6.1 , which is proved in the same section.

\section{§2. Properties of the operators $\widehat{d}_{ \pm}(k)$}

In this section we present the properties of $\widehat{d}_{ \pm}(k), k \in \mathbb{R}^{2}$, which were considered in detail in [29] and the proofs of which can be found in [28, 29]. In the case where $k=0$, we use the abbreviation $\widehat{d}_{ \pm} \doteq \widehat{d}_{ \pm}(0)$. The results of this section will be employed substantially in what follows.

Estimates (1.3) imply that the operators $\widehat{d}_{ \pm}(k), k \in \mathbb{R}^{2}$, are closed. If $k \notin 2 \pi \mathbb{Z}^{2}$, then $\operatorname{ker} \widehat{d}_{ \pm}(k)=\{0\}$ and $R\left(\widehat{d}_{ \pm}(k)\right)=L^{2}(K)$; $\operatorname{ker} \widehat{d}_{+}=\operatorname{ker} \widehat{d}_{-}$is the one-dimensional subspace of constant functions in $L^{2}(K)$, and the subspaces $R\left(\widehat{d}_{ \pm}\right)$are closed subspaces in $L^{2}(K)$ for which dim coker $\widehat{d}_{ \pm}=1$. For all $\varphi \in \widetilde{H}^{1}(K)$ we have

$$
\overline{\widehat{d}_{+} \varphi}=-\widehat{d}_{-} \bar{\varphi}
$$

(the bar stands for complex conjugation). 
On the set $\Gamma$ we consider the metric

$$
\begin{aligned}
& \rho_{\infty}\left(\{\mathcal{F}, \mathcal{G}, \mathcal{H}\},\left\{\mathcal{F}^{\prime}, \mathcal{G}^{\prime}, \mathcal{H}^{\prime}\right\}\right) \\
& \quad=\max \left\{\left\|\mathcal{F}-\mathcal{F}^{\prime}\right\|_{L^{\infty}(K)},\left\|\mathcal{G}-\mathcal{G}^{\prime}\right\|_{L^{\infty}(K)},\left\|\mathcal{H}-\mathcal{H}^{\prime}\right\|_{L^{\infty}(K)}\right\} .
\end{aligned}
$$

Let $\widehat{P}(\mathcal{L})$ denote the orthogonal projection in $L^{2}(K)$ onto the subspace $\mathcal{L}$; the set of orthogonal projections is endowed with the operator topology (induced by the operator norm).

Lemma 2.1 ([28]). The functions $\left(\Gamma(p, q, F), \rho_{\infty}\right) \ni\{\mathcal{F}, \mathcal{G}, \mathcal{H}\} \mapsto \widehat{P}\left(\right.$ coker $\left.\widehat{d}_{ \pm}\right)$are uniformly continuous.

This lemma and the convexity of the sets $\Gamma(p, q, F)$ imply that the following functions exist and are continuous:

$$
\left(\Gamma, \rho_{\infty}\right) \ni\{\mathcal{F}, \mathcal{G}, \mathcal{H}\} \mapsto \chi_{ \pm} \in\left\{\chi \in L^{2}(K):\|\chi\|=1\right\} \cap \operatorname{coker} \widehat{d}_{ \pm} \subset L^{2}(K),
$$

and we may assume that $\chi_{-}=\overline{\chi_{+}}($see $(2.1))$.

Lemma 2.2 (29). For any $\{\mathcal{F}, \mathcal{G}, \mathcal{H}\} \in \Gamma$ for a.e. $x \in K$ we have $\chi_{+}(x) \neq 0$.

Lemma $2.3(29])$. Let $\{\mathcal{F}, \mathcal{G}, \mathcal{H}\} \in \Gamma(p, q, F)$, and let $g($.$) be a positive, monotone$ nondecreasing, and continuously differentiable function on $(0,1]$ such that $g(r / 2) / g(r) \rightarrow$ 1 as $r \rightarrow+0$. Then for all $x \in \mathbb{R}^{2}$ and all $\Phi \in \widetilde{H}^{1}(K)$ we have

$$
\int_{y:|x-y| \leq 1} g(|x-y|)|\nabla \Phi(y)|_{\mathbb{C}^{2}}^{2} d^{2} y \leq c_{3} \int_{y:|x-y| \leq 1} g(|x-y|)\left|\widehat{d}_{+} \Phi(y)\right|^{2} d^{2} y,
$$

where $c_{3}=c_{3}(p, q, F ; g)>0$ (the integrals may take the value $+\infty$ ).

We denote by $\widetilde{H}_{0}^{1}\left\{g, \mathbb{Z}^{2}\right\}, g \in \mathbb{G}$, the Banach space of functions $\Phi \in \widetilde{H}_{0}^{1}(K)$ such that

$$
\|\Phi\|_{\widetilde{H}_{0}^{1}\left\{g, \mathbb{Z}^{2}\right\}} \doteq\||\nabla \Phi(.)|\|_{L^{2}\left\{g, \mathbb{Z}^{2}\right\}}<+\infty .
$$

By Lemma 2.3, $\widetilde{H}_{0}^{1}\left\{g, \mathbb{Z}^{2}\right\}=\left\{\Phi \in \widetilde{H}_{0}^{1}(K): \widehat{d}_{+} \Phi \in L^{2}\left\{g, \mathbb{Z}^{2}\right\}\right\}$. Since $r^{\varepsilon} g(r) \rightarrow 0$ as $r \rightarrow+0$ for any $\varepsilon>0$, the space $\widetilde{C}_{0}^{1}(K)$ is embedded continuously in $\widetilde{H}_{0}^{1}\left\{g, \mathbb{Z}^{2}\right\}$. On the other hand, $\widetilde{H}_{0}^{1}\left\{g, \mathbb{Z}^{2}\right\} \subset \widetilde{C_{0}}(K)$, and for all $\Phi \in \widetilde{H}_{0}^{1}\left\{g, \mathbb{Z}^{2}\right\}$ we have

$$
\|\Phi\|_{L^{\infty}(K)} \leq c_{4}\|\Phi\|_{\widetilde{H}_{0}^{1}\left\{g, \mathbb{Z}^{2}\right\}}
$$

with $c_{4}=c_{4}(g)>0$ (see 29]). The following Lemma 2.4 is an immediate consequence of Lemma 2.3 and estimate $(2.2)$.

Lemma $2.4\left([29)\right.$. If $\{\mathcal{F}, \mathcal{G}, \mathcal{H}\} \in \Gamma(p, q, F)$ and $g \in \mathbb{G}$, then there is a number $c_{5}=$ $c_{5}(p, q, F ; g)>0$ such that for any $\Phi \in \widetilde{H}_{0}^{1}(K)$ with $\widehat{d}_{+} \Phi \in L^{2}\left\{g, \mathbb{Z}^{2}\right\}$ we have $\Phi \in \widetilde{C}_{0}(K)$ and

$$
\|\Phi\|_{L^{\infty}(K)} \leq c_{5}\left\|\widehat{d}_{+} \Phi\right\|_{L^{2}\left\{g, \mathbb{Z}^{2}\right\}} .
$$

The next lemma follows from the definition of the set $\mathbb{L}\left(\mathbb{R}^{2}\right)$ (see, e.g., 22, 36]).

Lemma 2.5. Suppose $W \in \mathbb{L}\left(\mathbb{R}^{2}\right)$. Then for any $\varepsilon>0$ there exists a number $C_{\varepsilon}(W) \geq 0$ such that for all $k \in \mathbb{R}^{2}$ and all $\varphi \in \widetilde{H}^{1}(K)$ we have $W \varphi \in L^{2}(K)$ and

$$
\|W \varphi\|_{L^{2}(K)} \leq \varepsilon\|(k-i \nabla) \varphi\|_{L^{2}\left(K ; \mathbb{C}^{2}\right)}+C_{\varepsilon}(W)\|\varphi\|_{L^{2}(K)} .
$$

Theorem 2.1 ([29]). Suppose $\{\mathcal{F}, \mathcal{G}, \mathcal{H}\} \in \Gamma, g \in \mathbb{G}$. Then for any $\Phi \in \widetilde{H}_{0}^{1}(K)$ with $\widehat{d}_{+} \Phi \in L^{2}\left\{g, \mathbb{Z}^{2}\right\}$, and for any $\psi \in \widetilde{H}^{1}(K)$, we have $\Phi \in \widetilde{C}_{0}(K), e^{i \Phi} \psi \in \widetilde{H}^{1}(K)$, $\left(\partial \Phi / \partial x_{j}\right) \psi \in L^{2}(K), j=1,2$, and

$$
\widehat{d}_{+}\left(e^{i \Phi} \psi\right)=e^{i \Phi}\left(i\left(\widehat{d}_{+} \Phi\right) \psi+\widehat{d}_{+} \psi\right) .
$$


Theorem 2.1 is a consequence of Lemmas 2.3, 2.4, and 2.5. For the proof of identity (2.3), we use the fact that the operator $\widehat{d}_{+}$is closed. First, identity $(2.3)$ is established for $\psi \in \widetilde{C}^{1}(K)$, and then, with the use of Lemma 2.5, in the general case, for $\psi \in \widetilde{H}^{1}(K)$.

Theorem $2.2([28])$. Suppose $\{\mathcal{F}, \mathcal{G}, \mathcal{H}\} \in \Gamma$. Then there exist unique real-valued functions $\Phi, \Psi \in \widetilde{H}_{0}^{1}(K)$ and a vector $\widetilde{\varkappa} \in \mathbb{R}^{2}$ such that

$$
i \widehat{d}_{+}(\Phi-i \Psi)=-(\mathcal{G}+i \mathcal{F}) \widetilde{\varkappa}_{1}-i \mathcal{H}\left(\widetilde{\varkappa}_{2}+i\right) .
$$

Moreover, (2.4) implies that $\Phi, \Psi \in \widetilde{C}_{0}(K)$ and $\widetilde{\varkappa}_{1}>0$.

Lemma $2.6([29])$. Suppose $\{\mathcal{F}, \mathcal{G}, \mathcal{H}\} \in \Gamma$. Then $\chi_{+}=c_{6}(\mathcal{G H})^{-1}\left(\widehat{d}_{+} \Psi-\mathcal{H}\right)$, where $\Psi \in \widetilde{H}_{0}^{1}(K) \cap \widetilde{C}(K)$ is the function defined in Theorem 2.2 , and $c_{6}=c_{6}(\mathcal{F}, \mathcal{G}, \mathcal{H}) \in \mathbb{C} \backslash\{0\}$.

Lemma 2.7 29. Suppose $\{\mathcal{F}, \mathcal{G}, \mathcal{H}\} \in \Gamma$ and $\Psi \in \widetilde{H}_{0}^{1}(K)$ is the function defined in Theorem 2.2. Then

$$
\left(\frac{\partial \Psi}{\partial x_{1}}\right)^{2}+\left(\frac{\partial \Psi}{\partial x_{2}}-1\right)^{2}>0
$$

for a.e. $x \in K$.

Lemma 2.7 is a consequence of Lemmas 2.2 and 2.6.

Lemma 2.8 [29]. Under the conditions of Lemma 2.7, for all $\lambda \in \mathbb{R}$ we have

$$
\operatorname{meas}\left\{x \in K: \Psi(x)-x_{2}=\lambda\right\}=0 .
$$

Lemma 2.8 follows from Lemma 2.7 , because $\Psi \in H_{\text {loc }}^{1}\left(\mathbb{R}^{2}\right)$ and if $\Psi(x)-x_{2}=\lambda(=$ const) on some set $M \subseteq K$ with meas $M>0$, then $\partial \Psi / \partial x_{1}=0$ and $\partial \Psi / \partial x_{2}=1$ for a.e. $x \in M$, which contradicts Lemma 2.7. Lemma 2.8 will be used in the proof of Theorem 1.2. Instead of Lemma 2.8, we could apply Theorem 2.3 stated below (this method of argument was chosen in 28 for the proof of the absence of eigenvalues in the spectrum of the generalized two-dimensional periodic Dirac operator $\widehat{\mathcal{D}}+\widehat{V}$ with a matrix-valued potential $\left.\widehat{V} \in L_{\text {loc }}^{q}\left(\mathbb{R}^{2} ; \mathcal{M}_{2}\right), q>2\right)$.

Theorem 2.3 [28]. Suppose $\{\mathcal{F}, \mathcal{G}, \mathcal{H}\} \in \Gamma$ and identity (2.4) is fulfilled for real-valued functions $\Phi, \Psi \in \widetilde{H}_{0}^{1}(K) \cap \widetilde{C}(K)$ and a vector $\widetilde{\varkappa}=\left(\widetilde{\varkappa}_{1}, \widetilde{\varkappa}_{2}\right) \in \mathbb{R}^{2}$. Then $\mathbb{R}^{2} \ni x \mapsto$ $\mathcal{Z}(x)=\Phi(x)-i \Psi(x)+\tilde{\varkappa}_{1} x_{1}+\left(\tilde{\varkappa}_{2}+i\right) x_{2} \in \mathbb{C}$ is a continuous bijective map (with continuous inverse).

Under the conditions of Theorem $2.3, \mathcal{Z}($.$) is a periodic map: for any x \in \mathbb{R}^{2}$ and $n \in \mathbb{Z}^{2}$, we have $\mathcal{Z}(x+n)=\mathcal{Z}(x)+\widetilde{\varkappa}_{1} n_{1}+\left(\widetilde{\varkappa}_{2}+i\right) n_{2}$, and $\widetilde{\varkappa}_{1}>0$.

§3. Similarity between the Dirac operator $\widehat{\mathcal{D}}(0)+\widehat{V}$ with a special MatriX-VALUED POTENTIAL AND the DiRAC OPERATOR $\widehat{\mathcal{D}}(k+i \varkappa)$

We put

$$
\widetilde{H}_{0}^{1}\{\mathbb{G}\}=\bigcup_{g \in \mathbb{G}} \widetilde{H}_{0}^{1}\left\{g, \mathbb{Z}^{2}\right\} .
$$

Lemma 2.3 and Theorem 2.1 imply that for any $\Phi \in \widetilde{H}_{0}^{1}\{\mathbb{G}\}$ the operators of multiplication by $e^{\Phi}$ and by $e^{\widehat{\sigma}_{3} \Phi}$ act within the space $\widetilde{H}^{1}\left(K ; \mathbb{C}^{2}\right)$.

Theorem 3.1. Suppose $\{\mathcal{F}, \mathcal{G}, \mathcal{H}\} \in \Gamma(p, q, F)$ and $g \in \mathbb{G}$. For any two functions $\mathcal{C}_{1}, \mathcal{C}_{2} \in L^{2}\left\{g, \mathbb{Z}^{2}\right\}$ there exist unique vectors $k, \varkappa \in \mathbb{R}^{2}$ and functions $\Phi, \Psi \in \widetilde{H}_{0}^{1}\{\mathbb{G}\} \subset$ $\widetilde{H}_{0}^{1}(K) \cap \widetilde{C}(K)$ such that for some $\mu \in \mathbb{C} \backslash\{0\}$ (consequently, for all $\mu \in \mathbb{C}$ ) we have

$$
e^{\mu \widehat{\sigma}_{3} \Psi} e^{-i \mu \Phi} \widehat{\mathcal{D}}(\mu(k+i \varkappa)) e^{i \mu \Phi} e^{\mu \widehat{\sigma}_{3} \Psi}=\widehat{\mathcal{D}}(0)+\mu\left(\mathcal{C}_{1} \widehat{\sigma}_{1}+\mathcal{C}_{2} \widehat{\sigma}_{2}\right) .
$$


Moreover, $\Phi, \Psi \in \widetilde{H}_{0}^{1}\left\{g, \mathbb{Z}^{2}\right\}$ and

$$
\begin{aligned}
\max \left\{\|\Phi\|_{L^{\infty}(K)},\|\Psi\|_{L^{\infty}(K)}\right\} & \leq c_{1}^{\prime}\left(\left\|\mathcal{C}_{1}\right\|_{L^{2}\left\{g, \mathbb{Z}^{2}\right\}}+\left\|\mathcal{C}_{2}\right\|_{L^{2}\left\{g, \mathbb{Z}^{2}\right\}}\right), \\
|k|^{2}+|\varkappa|^{2} & \leq c_{2}^{\prime}\left(\left\|\mathcal{C}_{1}\right\|_{L^{2}(K)}^{2}+\left\|\mathcal{C}_{2}\right\|_{L^{2}(K)}^{2}\right),
\end{aligned}
$$

where $c_{1}^{\prime}=c_{1}^{\prime}(p, q, F ; g)>0, c_{2}^{\prime}=c_{2}^{\prime}(p, q, F)>0$. If $\mathcal{C}_{1} \pm i \mathcal{C}_{2} \in R\left(\widehat{d}_{ \pm}\right)$, then $k=\varkappa=0$. If $\mathcal{C}_{1}$ and $\mathcal{C}_{2}$ are real-valued, then $\varkappa=0$ and $\Phi$ and $\Psi$ are also real-valued.

Proof. By Theorem 2.1 and relations (1.2) and (2.1), vectors $k, \varkappa \in \mathbb{R}^{2}$ and functions $\Phi, \Psi \in \widetilde{H}_{0}^{1}\{\mathbb{G}\} \subset \widetilde{H}_{0}^{1}(K) \cap \widetilde{C}(K)$ satisfy (3.1) for some $\mu \in \mathbb{C} \backslash\{0\}$ (and hence, for all $\mu \in \mathbb{C})$ if and only if, for both signs + and - ,

$$
i \widehat{d}_{ \pm} \Phi_{ \pm}=\mathcal{C}_{ \pm}^{\prime} \doteq \mathcal{C}_{ \pm}-\left((\mathcal{G} \pm i \mathcal{F}) k_{1} \pm i \mathcal{H} k_{2}\right)-i\left((\mathcal{G} \pm i \mathcal{F}) \varkappa_{1} \pm i \mathcal{H} \varkappa_{2}\right),
$$

where $\Phi_{ \pm} \doteq \Phi \mp i \Psi, \mathcal{C}_{ \pm} \doteq \mathcal{C}_{1} \pm i \mathcal{C}_{2} \in L^{2}\left\{g, \mathbb{Z}^{2}\right\} \subset L^{2}(K)$ (multiplication by $e^{i \mu \Phi}$ and by $e^{\mu \Psi}, \mu \in \mathbb{C}$, acts within the space $\left.\widetilde{H}^{1}(K)\right)$. We denote $\left(\chi_{ \pm}, \mathcal{G} \pm i \mathcal{F}\right)=\mu_{ \pm}^{(1)}$, $\left(\chi_{ \pm}, \pm i \mathcal{H}\right)=\mu_{ \pm}^{(2)}$. Since the functions $\chi_{ \pm}$(with $\left\|\chi_{ \pm}\right\|=1$ ) are chosen so that $\chi_{-}=\overline{\chi_{+}}$, we have $\mu_{-}^{(1)}=\overline{\mu_{+}^{(1)}}, \mu_{-}^{(2)}=\overline{\mu_{+}^{(2)}}$. Also, $\left|\mu_{ \pm}^{(1)}\right| \leq p+F$ and $\left|\mu_{ \pm}^{(2)}\right| \leq p$. Since the subspaces $R\left(\widehat{d}_{ \pm}\right)$are closed in $L^{2}(K)$ and dimcoker $\widehat{d}_{ \pm}=1$, equations (3.4) can be solved for $\Phi_{ \pm} \in \widetilde{H}_{0}^{1}(K)$ and $k, \varkappa \in \mathbb{R}^{2}$ if and only if

$$
\left(\chi_{ \pm}, \mathcal{C}_{ \pm}\right)=\mu_{ \pm}^{(1)}\left(k_{1}+i \varkappa_{1}\right)+\mu_{ \pm}^{(2)}\left(k_{2}+i \varkappa_{2}\right) .
$$

Lemma 3.1. We have $\left|\operatorname{Im} \mu_{+}^{(1)} \overline{\mu_{+}^{(2)}}\right| \geq c_{0}=c_{0}(p, q, F)>0$.

Proof. Let $\mathcal{K}(x)=(\mathcal{G}(x) \mathcal{H}(x))^{-1 / 2}, x \in K$. For any vector $\tau=\left(\tau_{1}, \tau_{2}\right) \in \mathbb{R}^{2}$ and any function $\Omega_{+} \in \widetilde{H}^{1}(K)$, we can write

$$
\begin{aligned}
& \left\|\mathcal{K}\left(i \widehat{d}_{+} \Omega_{+}+(\mathcal{G}+i \mathcal{F}) \tau_{1}+i \mathcal{H} \tau_{2}\right)\right\|^{2} \\
& \quad=\left\|\mathcal{K} \mathcal{G}\left(\tau_{1}+\frac{\partial \Omega_{+}}{\partial x_{1}}\right)\right\|^{2}+\left\|\mathcal{K}\left(\mathcal{F}\left(\tau_{1}+\frac{\partial \Omega_{+}}{\partial x_{1}}\right)+\mathcal{H}\left(\tau_{2}+\frac{\partial \Omega_{+}}{\partial x_{2}}\right)\right)\right\|^{2},
\end{aligned}
$$

whence

$$
\begin{aligned}
& \left\|i \widehat{d}_{+} \Omega_{+}+(\mathcal{G}+i \mathcal{F}) \tau_{1}+i \mathcal{H} \tau_{2}\right\|^{2} \\
& \quad \geq c_{0} \sum_{j=1}^{2}\left\|\tau_{j}+\frac{\partial \Omega_{+}}{\partial x_{j}}\right\|^{2}=c_{0}\left(|\tau|^{2}+\sum_{j=1}^{2}\left\|\frac{\partial \Omega_{+}}{\partial x_{j}}\right\|^{2}\right),
\end{aligned}
$$

where $c_{0}=c_{0}(p, q, F)>0$. Since the function $\Omega_{+} \in \widetilde{H}^{1}(K)$ in (3.6) is arbitrary, we obtain

$$
\begin{aligned}
c_{0}|\tau|^{2} & \leq \min _{\Omega_{+} \in \widetilde{H}^{1}(K)}\left\|i \widehat{d}_{+} \Omega_{+}+(\mathcal{G}+i \mathcal{F}) \tau_{1}+i \mathcal{H} \tau_{2}\right\|^{2} \\
& =\left|\left(\chi_{+}, \mathcal{G}+i \mathcal{F}\right) \tau_{1}+\left(\chi_{+}, i \mathcal{H}\right) \tau_{2}\right|^{2}=\left|\mu_{+}^{(1)} \tau_{1}+\mu_{+}^{(2)} \tau_{2}\right|^{2}
\end{aligned}
$$

(in particular, this implies that $\left|\mu_{ \pm}^{(j)}\right| \geq \sqrt{c_{0}}, j=1,2$ ). Consequently,

$$
c_{0} \leq \sqrt{c_{0}}\left|\mu_{+}^{(2)}\right| \leq \min _{t \in \mathbb{R}}\left|\left(\mu_{+}^{(1)}+\mu_{+}^{(2)} t\right) \overline{\mu_{+}^{(2)}}\right|=\left|\operatorname{Im} \mu_{+}^{(1)} \overline{\mu_{+}^{(2)}}\right| .
$$

Lemma 3.1 is proved.

Since

$$
\operatorname{det}\left(\begin{array}{cc}
\mu_{+}^{(1)} & \mu_{+}^{(2)} \\
\mu_{-}^{(1)} & \mu_{-}^{(2)}
\end{array}\right)=2 i \operatorname{Im} \mu_{+}^{(1)} \overline{\mu_{+}^{(2)}},
$$


Lemma 3.1 shows that there exist unique vectors $k, \varkappa \in \mathbb{R}^{2}$ satisfying (3.5):

$$
\begin{aligned}
& k_{1}+i \varkappa_{1}=\left(2 i \operatorname{Im} \mu_{+}^{(1)} \overline{\mu_{+}^{(2)}}\right)^{-1}\left(\mu_{-}^{(2)}\left(\chi_{+}, \mathcal{C}_{+}\right)-\mu_{+}^{(2)}\left(\chi_{-}, \mathcal{C}_{-}\right)\right), \\
& k_{2}+i \varkappa_{2}=\left(2 i \operatorname{Im} \mu_{+}^{(1)} \overline{\mu_{+}^{(2)}}\right)^{-1}\left(-\mu_{-}^{(1)}\left(\chi_{+}, \mathcal{C}_{+}\right)+\mu_{+}^{(1)}\left(\chi_{-}, \mathcal{C}_{-}\right)\right) .
\end{aligned}
$$

Relations (3.7) and (3.8) imply (3.3). For the vectors $k, \varkappa \in \mathbb{R}^{2}$ chosen as above, we have $\mathcal{C}_{ \pm}^{\prime} \in R\left(\widehat{d}_{ \pm}\right)$; therefore, we can find (unique) functions $\Phi_{ \pm} \in \widetilde{H}_{0}^{1}(K)$ such that $i \widehat{d}_{ \pm} \Phi_{ \pm}=\mathcal{C}_{ \pm}^{\prime}$. On the other hand, $\mathcal{C}_{ \pm}^{\prime} \in L^{2}\left\{g, \mathbb{Z}^{2}\right\}$, so that, by Lemmas 2.3 and 2.4, $\Phi_{ \pm} \in \widetilde{H}_{0}^{1}\left\{g, \mathbb{Z}^{2}\right\} \subset \widetilde{C}_{0}(K)$ and

$$
\left\|\Phi_{ \pm}\right\|_{L^{\infty}(K)} \leq c_{5}\left\|\mathcal{C}_{ \pm}^{\prime}\right\|_{L^{2}\left\{g, \mathbb{Z}^{2}\right\}}
$$

Also, we have $\Phi, \Psi \in \widetilde{H}_{0}^{1}\left\{g, \mathbb{Z}^{2}\right\} \subset \widetilde{H}_{0}^{1}(K) \cap \widetilde{C}(K)$, and (3.2) is implied by (3.3), (3.4), and (3.9). If $\mathcal{C}_{ \pm} \in R\left(\widehat{d}_{ \pm}\right)$, then $\left(\chi_{ \pm}, \mathcal{C}_{ \pm}\right)=0$, whence $k=\varkappa=0$. If $\mathcal{C}_{1}$ and $\mathcal{C}_{2}$ are real-valued, then $\mathcal{C}_{-}=\overline{\mathcal{C}_{+}}$and $\left(\chi_{-}, \mathcal{C}_{-}\right)=\overline{\left(\chi_{+}, \mathcal{C}_{+}\right)}$, and from (3.7), (3.8) it follows that $\varkappa=0$ and $i \widehat{d}_{ \pm} \Phi_{ \pm}=\mathcal{C}_{ \pm}-\left((\mathcal{G} \pm i \mathcal{F}) k_{1} \pm i \mathcal{H} k_{2}\right)$ (see (3.4)). By complex conjugation and (2.1), we obtain $i \widehat{d}_{ \pm} \overline{\Phi_{\mp}}=\mathcal{C}_{ \pm}-\left((\mathcal{G} \pm i \mathcal{F}) k_{1} \pm i \mathcal{H} k_{2}\right)=i \widehat{d}_{ \pm} \Phi_{ \pm}$, whence $\widehat{d}_{+}(\operatorname{Im} \Phi-i \operatorname{Im} \Psi)=0$. Consequently, $\Phi$ and $\Psi$ are real-valued. This proves Theorem 3.1.

If under the assumptions of Theorem 3.1 we put $\mathcal{C}_{1}=i \mathcal{H}, \mathcal{C}_{2} \equiv 0$, then $\mathcal{C}_{1}, \mathcal{C}_{2} \in$ $L^{\infty}(K) \subset L^{2}\left\{g, \mathbb{Z}^{2}\right\}$ for any $g \in \mathbb{G}$. Therefore, the next theorem, which we need for what follows, is a consequence of Theorem 3.1.

Theorem 3.2. Suppose $\{\mathcal{F}, \mathcal{G}, \mathcal{H}\} \in \Gamma(p, q, F)$. The following objects exist and are unique: a vector $\widetilde{\varkappa} \in \mathbb{R}^{2}$ and real-valued functions $\Phi, \Psi \in \widetilde{H}_{0}^{1}\{\mathbb{G}\} \subset \widetilde{H}_{0}^{1}(K) \cap \widetilde{C}(K)$ such that for all $k, \varkappa \in \mathbb{R}^{2}$ and all $\mu \in \mathbb{R}$ we have

$$
e^{i \mu \widehat{\sigma}_{3} \Psi} e^{\mu \Phi} \widehat{\mathcal{D}}(k+i \varkappa+i \mu \tilde{\varkappa}) e^{-\mu \Phi} e^{i \mu \widehat{\sigma}_{3} \Psi}=\widehat{\mathcal{D}}(k+i \varkappa)+i \mu \mathcal{H} \widehat{\sigma}_{1} .
$$

Moreover, $\Phi, \Psi \in \widetilde{H}_{0}^{1}\left\{g, \mathbb{Z}^{2}\right\}$ for every $g \in \mathbb{G}$, and $\max \left\{\|\Phi\|_{L^{\infty}(K)},\|\Psi\|_{L^{\infty}(K)}\right\} \leq c_{1}^{*}$, $|\widetilde{\varkappa}| \leq c_{2}^{*}$, where $c_{1}^{*}=c_{1}^{*}(p, q, F)>0$ and $c_{2}^{*}=c_{2}^{*}(p, q, F)>0$.

The functions $\Phi, \Psi$ and the vector $\tilde{\varkappa}$ defined in Theorem 3.2 coincide with the corresponding objects in Theorem 2.2, because they satisfy condition (2.4), which is implied by $(3.10)$.

Lemma 3.2. For the vector $\tilde{\varkappa}=\left(\tilde{\varkappa}_{1}, \tilde{\varkappa}_{2}\right) \in \mathbb{R}^{2}$ defined in Theorem 3.2 we have $\tilde{\varkappa}_{1} \geq$ $c_{3}^{*}=c_{3}^{*}(p, q, F)>0$.

Proof. Identity (2.4) yields

$$
\mu_{+}^{(1)} \tilde{\varkappa}_{1}+\mu_{+}^{(2)}\left(\tilde{\varkappa}_{2}+i\right)=0 .
$$

Since $\left|\mu_{+}^{(1)}\right| \leq p+F$ and $\left|\mu_{+}^{(2)}\right| \geq \sqrt{c_{0}}$ (see the proof of Lemma 3.1), from (3.11) we deduce that

$$
\left|\tilde{\varkappa}_{1}\right| \geq \frac{\sqrt{c_{0}}}{p+F}\left|\operatorname{Im} \frac{\mu_{+}^{(1)}}{\mu_{+}^{(2)}} \tilde{\varkappa}_{1}\right|=\frac{\sqrt{c_{0}}}{p+F} \doteq c_{3}^{*},
$$

and $\tilde{\varkappa}_{1}>0$ by Theorem 2.2 .

\section{$\S 4$. Auxiliary statements}

Let $k \in \mathbb{R}^{2}, \mu \in \mathbb{R}$. For every $N \in \mathbb{Z}^{2}$, we denote

$$
\begin{aligned}
& G_{N}^{ \pm}(k ; \mu)=\left(\left(k_{1}+2 \pi N_{1}\right)^{2}+\left(k_{2}+2 \pi N_{2} \pm \mu\right)^{2}\right)^{1 / 2}, \\
& G_{N}(k ; \mu)=\min \left\{G_{N}^{-}(k ; \mu), G_{N}^{+}(k ; \mu)\right\}
\end{aligned}
$$


(here and in the sequel, we agree that the statements and formulas involving \pm and $\mp$ are understood independently for the upper and the lower combination of signs). If $k_{1}=\pi$, then $G_{N}(k ; \mu) \geq \pi$. For $\varphi \in \widetilde{H}^{1}(K)$, put

$$
\begin{aligned}
\|\varphi\|_{*} & =\left(\sum_{N \in \mathbb{Z}^{2}} G_{N}^{2}(k ; \mu)\left|\varphi_{N}\right|^{2}\right)^{1 / 2} \\
\|\varphi\|_{*, \pm} & =\left(\sum_{N \in \mathbb{Z}^{2}}\left(G_{N}^{ \pm}(k ; \mu)\right)^{2}\left|\varphi_{N}\right|^{2}\right)^{1 / 2} .
\end{aligned}
$$

For $a \geq 2 \pi$, we introduce the finite sets

$$
T^{ \pm}(a)=\left\{N \in \mathbb{Z}^{2}: G_{N}^{ \pm}(k ; \mu) \leq a\right\} .
$$

In the above notation, the dependence on the vector $k \in \mathbb{R}^{2}$ and the number $\mu \in \mathbb{R}$, which will be specified in advance, is not indicated explicitly. Let $\# \mathcal{O}$ denote the number of elements of a finite set $\mathcal{O}$. We have

$$
1 \leq \# T^{ \pm}(a)<6 \pi a^{2} .
$$

Lemma 4.1. Suppose $\{\mathcal{F}, \mathcal{G}, \mathcal{H}\} \in \Gamma(p, q, F)$. Then for all vectors $k \in \mathbb{R}^{2}$, all numbers $\mu \in \mathbb{R}$, and all functions $\varphi \in \widetilde{H}^{1}(K)$ we have

$$
c_{1}\|\varphi\|_{*, \pm}^{2} \leq\left\|\left(\widehat{d}_{ \pm}(k)+i \mu \mathcal{H}\right) \varphi\right\|^{2} \leq c_{2}\|\varphi\|_{*, \pm}^{2},
$$

where $c_{1}=c_{1}(p, q, F)>0$ and $c_{2}=c_{2}(p, q, F) \geq c_{1}$.

This lemma is a consequence of estimates (1.3) (with the same constants $c_{1}$ and $c_{2}$ ).

For a set $\mathcal{O} \subseteq \mathbb{Z}^{2}$, denote $\mathcal{L}(\mathcal{O})=\left\{\psi \in L^{2}(K): \psi_{N}=0\right.$ for $\left.N \in \mathbb{Z}^{2} \backslash \mathcal{O}\right\}, \mathcal{L}\left(\mathbb{Z}^{2}\right)=$ $L^{2}(K), \mathcal{L}(\varnothing)=\{0\}$. Let $\widehat{P}^{\mathcal{O}}=\widehat{P}(\mathcal{L}(\mathcal{O}))$ be the orthogonal projection in $L^{2}(K)$ that takes a function $\varphi \in L^{2}(K)$ to the function

$$
\widehat{P}^{\mathcal{O}} \varphi=\sum_{N \in \mathcal{O}} \varphi_{N} e^{2 \pi i(N, x)}
$$

Lemma 4.2. If $W \in L^{2}(K)$, then for any finite set $\mathcal{O} \subset \mathbb{Z}^{2}$ the operator $W \widehat{P}^{\mathcal{O}}$ is bounded on $L^{2}(K)$, and

$$
\left\|W \widehat{P}^{\mathcal{O}}\right\| \leq f_{W}(\# \mathcal{O}),
$$

where $f_{W}: \mathbb{Z}_{+} \rightarrow[0,+\infty)$ is a monotone nondecreasing function satisfying $f_{W}(N)=$ $o(\sqrt{N})$ as $N \rightarrow+\infty$.

Proof. For $b \geq 0$, we introduce the following functions:

$$
K \ni x \mapsto W_{b}(x)= \begin{cases}W(x) & \text { if }|W(x)|>b, \\ 0 & \text { otherwise }\end{cases}
$$

$$
\widetilde{W}_{b}(x)=W(x)-W_{b}(x), x \in K \text {. If } \varphi \in L^{2}(K) \text { and } \mathcal{O} \subset \mathbb{Z}^{2} \text { is a finite set, then }
$$

$$
\begin{aligned}
\left\|W_{b} \widehat{P}^{\mathcal{O}} \varphi\right\| & =\left(\sum_{N \in \mathbb{Z}^{2}}\left|\sum_{M \in \mathbb{Z}^{2}}\left(W_{b}\right)_{M}\left(\widehat{P}^{\mathcal{O}} \varphi\right)_{N-M}\right|^{2}\right)^{1 / 2} \\
& \leq\left(\sum_{N \in \mathbb{Z}^{2}}\left(\sum_{M: N-M \in \mathcal{O}}\left|\left(W_{b}\right)_{M}\right|^{2}\right)\left(\sum_{M: N-M \in \mathcal{O}}\left|\varphi_{N-M}\right|^{2}\right)\right)^{1 / 2} \\
& \leq\left(\sum_{M \in \mathbb{Z}^{2}}\left(\sum_{N: N-M \in \mathcal{O}} 1\right)\left|\left(W_{b}\right)_{M}\right|^{2}\right)^{1 / 2}\|\varphi\| \\
& =(\# \mathcal{O})^{1 / 2}\left\|W_{b}\right\|_{L^{2}(K)}\|\varphi\|,
\end{aligned}
$$


whence

$$
\left\|W \widehat{P}^{\mathcal{O}} \varphi\right\| \leq\left\|\widetilde{W}_{b} \widehat{P}^{\mathcal{O}} \varphi\right\|+\left\|W_{b} \widehat{P}^{\mathcal{O}} \varphi\right\| \leq\left(b+(\# \mathcal{O})^{1 / 2}\left\|W_{b}\right\|_{L^{2}(K)}\right)\|\varphi\|
$$

Put

$$
f_{W}(N)=\inf _{b \geq 0}\left(b+\sqrt{N}\left\|W_{b}\right\|_{L^{2}(K)}\right), \quad N \in \mathbb{Z}_{+}=\mathbb{N} \cup\{0\} .
$$

Then $\left\|W \widehat{P}^{\mathcal{O}}\right\| \leq f_{W}(\# \mathcal{O})$, the function $f_{W}$ is monotone nondecreasing, and for any $\varepsilon>0$ we can find a number $b(\varepsilon)>0$ such that $\left\|W_{b(\varepsilon)}\right\|_{L^{2}(K)}<\varepsilon$, so that $f_{W}(N) / \sqrt{N} \rightarrow 0$ as $N \rightarrow+\infty$.

Let $W \in \mathbb{L}\left(\mathbb{R}^{2}\right)$; we put

$$
h_{W}(t)=\inf _{\varepsilon>0}\left(\varepsilon+t^{-1} C_{\varepsilon}(W)\right), \quad t>0,
$$

where $C_{\varepsilon}(W)$ is as in Lemma 2.5. The function $h_{W}$ is monotone nondecreasing, and $h_{W}(t) \rightarrow 0$ as $t \rightarrow+\infty$.

Lemma 4.3. Suppose $W \in \mathbb{L}\left(\mathbb{R}^{2}\right), \mu \geq 4 \pi$. Then for all $k \in \mathbb{R}^{2}$ with $k_{1}=\pi$ and all $\varphi \in \mathcal{L}\left(T^{ \pm}(\mu / 2)\right)$ we have

$$
\|W \varphi\| \leq c_{7}\|\varphi\|_{*, \pm}=c_{7}\|\varphi\|_{*},
$$

where $c_{7}=c_{7}(W)>0$. If $2 \pi \leq a \leq \mu / 2$, then

$$
\|W \varphi\| \leq h_{W}(a)\|\varphi\|_{*}
$$

for all $k \in \mathbb{R}^{2}$ and all $\varphi \in \mathcal{L}\left(T^{ \pm}(\mu / 2) \backslash T^{ \pm}(a)\right)$.

Proof. By Lemma 2.5 (with $\varepsilon=1$ ), for all $\mu \in \mathbb{R}$, all $k \in \mathbb{R}^{2}$, and all $\varphi \in \widetilde{H}^{1}(K)$ we have

$$
\|W \varphi\| \leq\|\varphi\|_{*, \pm}+C_{1}(W)\|\varphi\| .
$$

On the other hand, if $\varphi \in \mathcal{L}\left(T^{ \pm}(\mu / 2)\right), \mu \geq 4 \pi$, then $\|\varphi\|_{*, \pm}=\|\varphi\|_{*}$, and $\|\varphi\|_{*} \geq \pi\|\varphi\|$ whenever $k_{1}=\pi$. Therefore, (4.5) implies (4.3) with $c_{7}=1+\pi^{-1} C_{1}(W)$. Now, suppose that $2 \pi \leq a \leq \mu / 2$ and $\varphi \in \mathcal{L}\left(T^{ \pm}(\mu / 2) \backslash T^{ \pm}(a)\right)$. Then, for any $k \in \mathbb{R}^{2}$, we have $\|\varphi\|_{*, \pm} \geq a\|\varphi\|$, and by Lemma 2.5 we obtain

$$
\|W \varphi\| \leq\left(\varepsilon+a^{-1} C_{\varepsilon}(W)\right)\|\varphi\|_{*}
$$

for any $\varepsilon>0$, which yields (4.4).

Lemma 4.4. Suppose $W \in \mathbb{L}\left(\mathbb{R}^{2}\right), \mu \geq 4 \pi$. Then for all $k \in \mathbb{R}^{2}$ and all $\varphi \in \widetilde{H}^{1}(K) \cap$ $\mathcal{L}\left(\mathbb{Z}^{2} \backslash\left(T^{+}(\mu / 2) \cup T^{-}(\mu / 2)\right)\right)$ we have

$$
\|W \varphi\| \leq 3 h_{W}(\mu)\|\varphi\|_{*} .
$$

This lemma follows from Lemma 2.5, since, under the assumptions of Lemma 4.4, we have $\|\varphi\| \leq 2 \mu^{-1}\|\varphi\|_{*}$ and $\|(k-i \nabla) \varphi\|_{L^{2}\left(K ; \mathbb{C}^{2}\right)} \leq 3\|\varphi\|_{*}$.

Lemma 4.5. Suppose $W \in L^{2}(K), \mu>4 \pi$, and $2 \pi \leq a<a^{\prime} \leq \mu / 2$. Then for all $\varphi \in \mathcal{L}\left(\mathbb{Z}^{2} \backslash T^{ \pm}\left(a^{\prime}\right)\right)$ and all $\psi \in \mathcal{L}\left(T^{ \pm}(a)\right)$ we have

$$
|(\varphi, W \psi)| \leq \sqrt{6 \pi} a\left(\sum_{N \in \mathbb{Z}^{2}: 2 \pi|N|>a^{\prime}-a}\left|W_{N}\right|^{2}\right)^{1 / 2}\|\varphi\|_{L^{2}(K)}\|\psi\|_{L^{2}(K)} .
$$


Proof. Indeed,

$$
\begin{aligned}
|(\varphi, W \psi)| & \leq \sum_{M \in \mathbb{Z}^{2}}\left|\varphi_{M}\right| \sum_{N \in \mathbb{Z}^{2}}\left|W_{N} \psi_{M-N}\right| \\
& \leq\|\varphi\|_{L^{2}(K)}\left(\sum_{M \in \mathbb{Z}^{2} \backslash T^{ \pm}\left(a^{\prime}\right)}\left(\sum_{N \in \mathbb{Z}^{2}}\left|W_{N} \psi_{M-N}\right|\right)^{2}\right)^{1 / 2} \\
& \leq\left(\sum_{N \in \mathbb{Z}^{2}}\left(\sum_{M: M \notin T} 1\right)\left|W_{N}\right|^{2}\right)^{1 / 2}\|\varphi\|_{L^{2}(K), M-N \in T^{ \pm}(a)}\|\psi\|_{L^{2}(K)} \\
& \leq \sqrt{6 \pi} a\left(\sum_{N \in \mathbb{Z}^{2}: 2 \pi|N|>a^{\prime}-a}\left|W_{N}\right|^{2}\right)^{1 / 2}\|\varphi\|_{L^{2}(K)}\|\psi\|_{L^{2}(K)}
\end{aligned}
$$

(we have used estimate (4.1)).

Lemma 4.6. For $W \in \mathbb{L}\left(\mathbb{R}^{2}\right)$, let $W_{b}, b \geq 0$, be the functions defined in (4.2). There exists a monotone nondecreasing function $\tilde{h}_{W}:[0,+\infty) \rightarrow[0,+\infty)$ such that $\tilde{h}_{W}(t) \rightarrow 0$ as $t \rightarrow+\infty$ and for all $\mu \in \mathbb{R}$, all $k \in \mathbb{R}^{2}$ with $k_{1}=\pi$, all $\varphi \in \widetilde{H}^{1}(K)$, and all $b \geq 0$ we have

$$
\left\|W_{b} \varphi\right\| \leq \tilde{h}_{W}(b)\|\varphi\|_{*, \pm} .
$$

Proof. By Lemma 2.5, for any $\varepsilon>0$ there exists a number $C_{\varepsilon}(W) \geq 0$ such that

$$
\|W \psi\| \leq \varepsilon\|\psi\|_{*, \pm}+C_{\varepsilon}(W)\|\psi\|
$$

for all $\mu \in \mathbb{R}, k \in \mathbb{R}^{2}$, and $\psi \in \widetilde{H}^{1}(K)$. We define

$$
\tilde{h}_{W}(b)=\inf _{\varepsilon>0} \min _{a \geq 2 \pi}\left(\sqrt{\frac{6}{\pi}} a\left\|W_{b}\right\|_{L^{2}(K)}+\varepsilon+a^{-1} C_{\varepsilon}(W)\right), \quad b \geq 0 .
$$

Since the function $[0,+\infty) \ni b \mapsto\left\|W_{b}\right\|_{L^{2}(K)}$ is monotone nondecreasing, and $\left\|W_{b}\right\|_{L^{2}(K)}$ $\rightarrow 0$ as $b \rightarrow+\infty$, the function $\widetilde{h}_{W}$ possesses the same properties. On the other hand, using (4.1) and (4.7), we see that if $\mu \in \mathbb{R}, k \in \mathbb{R}^{2}, k_{1}=\pi, \varphi \in \widetilde{H}^{1}(K)$ (in which case $\left.\pi\|\varphi\| \leq\|\varphi\|_{*, \pm}\right), b \geq 0, \varepsilon>0$ and $a \geq 2 \pi$, then

$$
\begin{aligned}
\left\|W_{b} \varphi\right\| \leq & \left\|W_{b} \widehat{P}^{T^{ \pm}(a)} \varphi\right\|+\left\|W_{b} \widehat{P}^{\mathbb{Z}^{2} \backslash T^{ \pm}(a)} \varphi\right\| \\
\leq & \left\|W_{b}\right\|_{L^{2}(K)}\left\|\widehat{P}^{T^{ \pm}(a)} \varphi\right\|_{L^{\infty}(K)}+\left\|W \widehat{P}^{\mathbb{Z}^{2} \backslash T^{ \pm}(a)} \varphi\right\| \\
\leq & \sqrt{6 \pi} a\left\|W_{b}\right\|_{L^{2}(K)}\left\|\widehat{P}^{T^{ \pm}(a)} \varphi\right\| \\
& +\varepsilon\left\|\widehat{P}^{\mathbb{Z}^{2} \backslash T^{ \pm}(a)} \varphi\right\|_{*, \pm}+C_{\varepsilon}(W)\left\|\widehat{P}^{\mathbb{Z}^{2} \backslash T^{ \pm}(a)} \varphi\right\| \\
\leq & \left(\sqrt{\frac{6}{\pi}} a\left\|W_{b}\right\|_{L^{2}(K)}+\varepsilon+a^{-1} C_{\varepsilon}(W)\right)\|\varphi\|_{*, \pm} .
\end{aligned}
$$

These inequalities and the definition of $\tilde{h}_{W}$ imply (4.6).

\section{$\S 5$. Proof of Theorem 1.2}

For an arbitrary set $\mathbf{M}^{\prime} \subseteq \mathbb{N}$ we put

$$
\mathcal{Q}\left(\mathbf{M}^{\prime}\right)=\varlimsup_{N \rightarrow+\infty} \frac{\#\left\{n \in \mathbf{M}^{\prime}: n \leq N\right\}}{N} .
$$

In this and the next sections we use the symbol $\sum_{+,-}$to denote the sum of two terms obtained from expressions with indices \pm and $\mp$ when fixing the upper or the lower combination of signs. 
Theorem 5.1. Suppose $\{\mathcal{F}, \mathcal{G}, \mathcal{H}\} \in \Gamma(p, q, F), \widetilde{V}^{(l)} \in \mathbb{L}\left(\mathbb{R}^{2}\right), l=0,3$, and $\Psi$ is a real-valued function of class $\widetilde{C}(K)$ such that

$$
\operatorname{meas}\left\{x \in K: \Psi(x)-x_{2}=\lambda\right\}=0
$$

for any $\lambda \in \mathbb{R}$. Then there exists a number $a_{0}=a_{0}\left(p, q, F ; \widetilde{V}^{(0)}, \widetilde{V}^{(3)}\right) \geq 2 \pi$ such that for any $a \geq a_{0}$ there is a set $\mathbf{M} \subset \mathbb{N}$, depending also on $\mathcal{F}, \mathcal{G}, \mathcal{H}, \widetilde{V}^{(0)}, \widetilde{V}^{(3)}$, and $\Psi$, for which $\mathcal{Q}(\mathbb{N} \backslash \mathbf{M})=0$ and for all $\mu \in \pi \mathbf{M}$, all $k \in \mathbb{R}^{2}$ with $k_{1}=\pi$, and all

$$
\varphi=\left(\begin{array}{c}
\varphi_{+} \\
\varphi_{-}
\end{array}\right) \in \widetilde{H}^{1}\left(K ; \mathbb{C}^{2}\right)
$$

we have the estimate

$$
\begin{aligned}
& \left\|\left(\widehat{\mathcal{D}}(k)+i \mu \mathcal{H} \widehat{\sigma}_{1}+e^{2 i \mu \widehat{\sigma}_{3} \Psi}\left(\widetilde{V}^{(0)} \widehat{I}+\widetilde{V}^{(3)} \widehat{\sigma}_{3}\right)\right) \varphi\right\|^{2} \\
& \quad \geq \frac{c_{1}}{6} \sum_{+,-}\left\|\widehat{P}^{T^{ \pm}(a)} \varphi_{ \pm}\right\|_{*}^{2}+c_{8} \sum_{+,-}\left\|\widehat{P}^{\mathbb{Z}^{2} \backslash T^{ \pm}(a)} \varphi_{ \pm}\right\|_{*, \pm}^{2}
\end{aligned}
$$

where $c_{8}=c_{8}\left(p, q, F ; \widetilde{V}^{(0)}, \widetilde{V}^{(3)}\right) \in\left(0, \frac{1}{6} c_{1}\right]$.

The proof of this theorem is postponed until $\S 6$.

We pass to the proof of Theorem 1.2, in which Theorems 3.1, 3.2, and 5.1 will play an important part. For $l=1,2$ and $b \geq 0$ (as in (4.2)), we introduce the functions

$$
\mathbb{R}^{2} \ni x \mapsto V_{b}^{(l)}(x)= \begin{cases}V^{(l)}(x) & \text { if }\left|V^{(l)}(x)\right|>b, \\ 0 & \text { otherwise. }\end{cases}
$$

Since $V^{(l)} \in \mathbb{L}\left(\mathbb{R}^{2}\right), l=1,2$, Lemma 4.6 allows us to choose a number $b=b\left(c_{1} ; V^{(1)}, V^{(2)}\right)$ $\geq 0$ so that for all $\mu \in \mathbb{R}$, all $k \in \mathbb{R}^{2}$ with $k_{1}=\pi$, and all $\psi \in \widetilde{H}^{1}(K)$ we have the inequalities

$$
\left\|V_{b}^{(l)} \psi\right\|^{2} \leq \frac{c_{1}}{192}\|\psi\|_{*, \pm}^{2}, \quad l=1,2
$$

for both signs + and - . For $l=1,2$ we have $\left\|V^{(l)}-V_{b}^{(l)}\right\|_{L^{\infty}\left(\mathbb{R}^{2}\right)} \leq b<+\infty$; therefore, by Theorem 3.1, there exist vectors $k^{\prime}, \varkappa^{\prime} \in \mathbb{R}^{2}$ and functions $\Phi^{\prime}, \Psi^{\prime} \in \widetilde{H}_{0}^{1}(K) \cap \widetilde{C}(K)$ with the following properties: the operators of multiplication by the functions $e^{ \pm i \Phi^{\prime}}$ and by the matrix-valued functions $e^{ \pm \widehat{\sigma}_{3} \Psi^{\prime}}$ act within the space $\widetilde{H}^{1}\left(K ; \mathbb{C}^{2}\right)$; for every $k, \varkappa \in \mathbb{R}^{2}$ we have

$$
\begin{array}{r}
e^{\widehat{\sigma}_{3} \Psi^{\prime}} e^{-i \Phi^{\prime}}\left(\widehat{\mathcal{D}}\left(k+k^{\prime}+i\left(\varkappa+\varkappa^{\prime}\right)\right)+\widehat{V}\right) e^{i \Phi^{\prime}} e^{\widehat{\sigma}_{3} \Psi^{\prime}} \\
=\widehat{\mathcal{D}}(k+i \varkappa)+\widetilde{V}^{(0)} \widehat{I}+\sum_{l=1}^{2} V_{b}^{(l)} \widehat{\sigma}_{l}+\widetilde{V}^{(3)} \widehat{\sigma}_{3},
\end{array}
$$

where

$$
\tilde{V}^{(0)}=V^{(0)} \cosh 2 \Psi^{\prime}+V^{(3)} \sinh 2 \Psi^{\prime}, \quad \widetilde{V}^{(3)}=V^{(0)} \sinh 2 \Psi^{\prime}+V^{(3)} \cosh 2 \Psi^{\prime},
$$

and

$$
\max \left\{\left\|\Phi^{\prime}\right\|_{L^{\infty}(K)},\left\|\Psi^{\prime}\right\|_{L^{\infty}(K)}\right\} \leq c_{1}^{\prime \prime} b,
$$

where $c_{1}^{\prime \prime}=c_{1}^{\prime \prime}(p, q, F)>0$. Inequality (5.6) shows that $\widetilde{V}^{(0)}, \widetilde{V}^{(3)} \in \mathbb{L}\left(\mathbb{R}^{2}\right)$. Let $\Phi, \Psi \in$ $\widetilde{H}_{0}^{1}(K) \cap \widetilde{C}(K)$ and $\widetilde{\varkappa} \in \mathbb{R}^{2}$ be the vector-valued functions and the vector defined in 
Theorem 3.2 (for the functions $\mathcal{F}, \mathcal{G}, \mathcal{H}$ ). Multiplications by $e^{\mu \Phi}$ and by $e^{i \mu \widehat{\sigma}_{3} \Psi}, \mu \in \mathbb{R}$, also act within the space $\widetilde{H}^{1}\left(K ; \mathbb{C}^{2}\right)$. From (3.10) we obtain

$$
\begin{gathered}
e^{i \mu \widehat{\sigma}_{3} \Psi} e^{\mu \Phi}\left(\widehat{\mathcal{D}}(k+i \mu \widetilde{\varkappa})+\widetilde{V}^{(0)} \widehat{I}+\sum_{l=1}^{2} V_{b}^{(l)} \widehat{\sigma}_{l}+\widetilde{V}^{(3)} \widehat{\sigma}_{3}\right) e^{-\mu \Phi} e^{i \mu \widehat{\sigma}_{3} \Psi} \\
=\widehat{\mathcal{D}}(k)+i \mu \mathcal{H} \widehat{\sigma}_{1}+\sum_{l=1}^{2} V_{b}^{(l)} \widehat{\sigma}_{l}+e^{2 i \mu \widehat{\sigma}_{3} \Psi}\left(\widetilde{V}^{(0)} \widehat{I}+\widetilde{V}^{(3)} \widehat{\sigma}_{3}\right)
\end{gathered}
$$

for all $\mu \in \mathbb{R}$ and all $k \in \mathbb{R}^{2}$. By Lemma 2.8, $\Psi$ satisfies (5.1). Let $a_{0}$ and $c_{8}$ be the numbers defined in Theorem 5.1 (for the functions $\widetilde{V}^{(0)}, \widetilde{V}^{(3)}$, and $\Psi$ ). We put $\varepsilon=\frac{1}{16} \sqrt{2 c_{8}}$ and choose a number $a \geq a_{0}$ so that $\varepsilon a \geq C_{\varepsilon}\left(V^{(l)}\right), l=1,2$, where $C_{\varepsilon}($.$) is$ as in (4.7). By Theorem 5.1, there exists a set $\mathbf{M} \subset \mathbb{N}$, depending on $\mathcal{F}, \mathcal{G}, \mathcal{H}$, on the matrix-valued potential $\widehat{V}$, and also on the choice of $b$ and $a$, such that $\mathcal{Q}(\mathbb{N} \backslash \mathbf{M})=0$ and estimate (5.3) is valid for all $\mu \in \pi \mathbf{M}$, all $k \in \mathbb{R}^{2}$ with $k_{1}=\pi$, and all vector-valued functions (5.2). Using (4.7), (5.4), and the estimates

$$
\left\|\widehat{P}^{\mathbb{Z}^{2} \backslash T^{ \pm}(a)} \varphi_{ \pm}\right\| \leq \frac{1}{a}\left\|\widehat{P}^{\mathbb{Z}^{2} \backslash T^{ \pm}(a)} \varphi_{ \pm}\right\|_{*, \pm},
$$

we obtain the inequalities

$$
\begin{aligned}
& \left\|\sum_{l=1}^{2} V_{b}^{(l)} \widehat{\sigma}_{l} \varphi\right\|^{2} \leq 2 \sum_{l=1}^{2}\left\|V_{b}^{(l)} \varphi\right\|^{2} \\
& \quad \leq 4 \sum_{l=1}^{2} \sum_{+,-}\left\|V_{b}^{(l)} \widehat{P}^{T^{ \pm}(a)} \varphi_{ \pm}\right\|^{2}+4 \sum_{l=1}^{2} \sum_{+,-}\left\|V^{(l)} \widehat{P}^{\mathbb{Z}^{2} \backslash T^{ \pm}(a)} \varphi_{ \pm}\right\|^{2} \\
& \quad \leq \frac{c_{1}}{24} \sum_{+,-}\left\|\widehat{P}^{T^{ \pm}(a)} \varphi_{ \pm}\right\|_{*, \pm}^{2}+8 \sum_{l=1}^{2} \sum_{+,-}\left(\varepsilon^{2}\left\|\widehat{P}^{\mathbb{Z}^{2} \backslash T^{ \pm}(a)} \varphi_{ \pm}\right\|_{*, \pm}^{2}+C_{\varepsilon}^{2}\left(V^{(l)}\right)\left\|\widehat{P}^{\mathbb{Z}^{2} \backslash T^{ \pm}(a)} \varphi_{ \pm}\right\|^{2}\right) \\
& \quad \leq \frac{c_{1}}{24} \sum_{+,-}\left\|\widehat{P}^{T^{ \pm}(a)} \varphi_{ \pm}\right\|_{*, \pm}^{2}+\frac{c_{8}}{4} \sum_{+,-}\left\|\widehat{P}^{\mathbb{Z}^{2} \backslash T^{ \pm}(a)} \varphi_{ \pm}\right\|_{*, \pm}^{2} \cdot
\end{aligned}
$$

Therefore, (5.3) implies that, again for all $\mu \in \pi \mathbf{M}$, all $k \in \mathbb{R}^{2}$ with $k_{1}=\pi$, and all $\varphi$ as in $(5.2)$,

$$
\begin{aligned}
\|(\widehat{\mathcal{D}}(k) & \left.+i \mu \mathcal{H} \widehat{\sigma}_{1}+\sum_{l=1}^{2} V_{b}^{(l)} \widehat{\sigma}_{l}+e^{2 i \mu \widehat{\sigma}_{3} \Psi}\left(\widetilde{V}^{(0)} \widehat{I}+\widetilde{V}^{(3)} \widehat{\sigma}_{3}\right)\right) \varphi \|^{2} \\
\geq & \frac{1}{2}\left\|\left(\widehat{\mathcal{D}}(k)+i \mu \mathcal{H} \widehat{\sigma}_{1}+e^{2 i \mu \widehat{\sigma}_{3} \Psi}\left(\widetilde{V}^{(0)} \widehat{I}+\widetilde{V}^{(3)} \widehat{\sigma}_{3}\right)\right) \varphi\right\|^{2}-\left\|\sum_{l=1}^{2} V_{b}^{(l)} \widehat{\sigma}_{l} \varphi\right\|^{2} \\
\geq & \frac{c_{1}}{24} \sum_{+,-}\left\|\widehat{P}^{T^{ \pm}(a)} \varphi_{ \pm}\right\|_{*, \pm}^{2}+\frac{c_{8}}{4} \sum_{+,-}\left\|\widehat{P}^{\mathbb{Z}^{2} \backslash T^{ \pm}(a)} \varphi_{ \pm}\right\|_{*, \pm}^{2} \\
\geq & \frac{c_{8}}{4} \sum_{+,-}\left\|\varphi_{ \pm}\right\|_{*, \pm}^{2} \geq \frac{c_{8}}{4} \sum_{N \in \mathbb{Z}^{2}} G_{N}^{2}(k ; \mu)\left|\varphi_{N}\right|^{2} \geq \frac{\pi^{2}}{4} c_{8}\|\varphi\|^{2} .
\end{aligned}
$$

Now we use (5.5) with $\varkappa=\mu \tilde{\varkappa}$ and also (5.6)-(5.8) and the estimate $\|\Phi\|_{L^{\infty}(K)} \leq c_{1}^{*}$ (see Theorem 3.2) to obtain the inequality

$$
\left\|\left(\widehat{\mathcal{D}}\left(k+k^{\prime}+i\left(\mu \widetilde{\varkappa}+\varkappa^{\prime}\right)\right)+\widehat{V}\right) \varphi\right\| \geq \frac{\pi}{2} \sqrt{c_{8}} e^{-4 c_{1}^{\prime \prime} b} e^{-2 c_{1}^{*} \mu}\|\varphi\|,
$$

which is valid for all $\mu \in \pi \mathbf{M}$, all $k \in \mathbb{R}^{2}$ with $k_{1}=\pi$, and all $\varphi \in \widetilde{H}^{1}\left(K ; \mathbb{C}^{2}\right)$. To complete the proof of Theorem 1.2 it remains to put $e=\tilde{\varkappa} /|\tilde{\varkappa}|$ and $c=3 c_{1}^{*} / c_{3}^{*}$ (see 
Lemma 3.2), and it suffices to choose numbers $\widetilde{\mu} \doteq|\tilde{\varkappa}| \mu \in \pi|\widetilde{\varkappa}| \mathbf{M}$ for which

$$
4 c_{1}^{\prime \prime} b-\ln \left(\frac{\pi}{2} \sqrt{c_{8}}\right) \leq c_{1}^{*} \frac{\tilde{\mu}}{|\widetilde{\varkappa}|} .
$$

Theorem 1.2 is proved.

Remark. Under the conditions of Theorem 1.2, if $V^{(l)} \in L^{2}\left\{g, \mathbb{Z}^{2}\right\}, l=1,2$, for some $g \in \mathbb{G}$, then the proof of Theorem 1.2 simplifies, because Theorem 3.1 provides an identity similar to (5.5) but without the term $\sum_{l=1}^{2} V_{b}^{(l)} \widehat{\sigma}_{l}$ on the right-hand side (see also [29]).

\section{§6. Proof of Theorem 5.1}

Under the conditions of Theorem 5.1, denote $V^{( \pm)}=\widetilde{V}^{(0)} \pm \widetilde{V}^{(3)}$. Since

$$
\widehat{\mathcal{D}}(k)+i \mu \mathcal{H} \widehat{\sigma}_{1}+e^{2 i \mu \widehat{\sigma}_{3} \Psi}\left(\widetilde{V}^{(0)} \widehat{I}+\widetilde{V}^{(3)} \widehat{\sigma}_{3}\right)=\left(\begin{array}{cc}
e^{2 i \mu \Psi} V^{(+)} & \widehat{d}_{-}(k)+i \mu \mathcal{H} \\
\widehat{d}_{+}(k)+i \mu \mathcal{H} & e^{-2 i \mu \Psi} V^{(-)}
\end{array}\right),
$$

Theorem 5.1 is equivalent to the following statement.

Theorem 6.1. Suppose $\{\mathcal{F}, \mathcal{G}, \mathcal{H}\} \in \Gamma(p, q, F), V^{( \pm)} \in \mathbb{L}\left(\mathbb{R}^{2}\right)$, and $\Psi$ is a real-valued function of class $\widetilde{C}(K)$ satisfying (5.1) for all $\lambda \in \mathbb{R}$. Then there exists a number $a_{0}^{\prime}=$ $a_{0}^{\prime}\left(p, q, F ; V^{(+)}, V^{(-)}\right) \geq 2 \pi$ with the following property: for every $a_{1} \geq a_{0}^{\prime}$ we can find a set $\mathbf{M} \subset \mathbb{N}$, depending also on $\mathcal{F}, \mathcal{G}, \mathcal{H}, V^{(+)}, V^{(-)}$, and $\Psi$, such that $\mathcal{Q}(\mathbb{N} \backslash \mathbf{M})=0$ and for all $\mu \in \pi \mathbf{M}$, all $k \in \mathbb{R}^{2}$ with $k_{1}=\pi$, and all $\varphi_{ \pm} \in \widetilde{H}^{1}(K)$ we have

$$
\begin{aligned}
\|\left(\widehat{d}_{+}(k)\right. & +i \mu \mathcal{H}) \varphi_{+}+e^{-2 i \mu \Psi} V^{(-)} \varphi_{-} \|^{2} \\
& +\left\|\left(\widehat{d}_{-}(k)+i \mu \mathcal{H}\right) \varphi_{-}+e^{2 i \mu \Psi} V^{(+)} \varphi_{+}\right\|^{2} \\
\geq & \frac{c_{1}}{6}\left(\left\|\widehat{P}^{T^{+}\left(a_{1}\right)} \varphi_{+}\right\|_{*}^{2}+\left\|\widehat{P}^{T^{-}\left(a_{1}\right)} \varphi_{-}\right\|_{*}^{2}\right) \\
& +c_{8}^{\prime}\left(\left\|\widehat{P}^{\mathbb{Z}^{2} \backslash T^{+}\left(a_{1}\right)} \varphi_{+}\right\|_{*,+}^{2}+\left\|\widehat{P}^{\mathbb{Z}^{2} \backslash T^{-}\left(a_{1}\right)} \varphi_{-}\right\|_{*,-}^{2}\right),
\end{aligned}
$$

where $c_{8}^{\prime}=c_{8}^{\prime}\left(p, q, F ; V^{(+)}, V^{(-)}\right) \in\left(0, \frac{1}{6} c_{1}\right]$.

The next lemma is a version of the Wiener theorem (see, e.g., 45, Theorem XI.114 and the remark after it]).

Lemma 6.1. Suppose $W \in L^{1}(K)$, and $\Psi$ is a real-valued function of class $\widetilde{C}(K)$ satisfying (5.1) for all $\lambda \in \mathbb{R}$. Then

$$
\lim _{N \rightarrow+\infty} \frac{1}{N} \sum_{\nu=1}^{N}\left|\int_{K} e^{2 \pi i \nu\left(\Psi-x_{2}\right)} W d^{2} x\right|^{2}=0 .
$$

Corollary. Under the conditions of Lemma 6.1, denote

$$
\mathbf{M}_{ \pm}(W, \Psi ; \theta)=\left\{\nu \in \mathbb{N}:\left|\int_{K} e^{ \pm 2 \pi i \nu\left(\Psi-x_{2}\right)} W d^{2} x\right| \geq \theta\right\}, \quad \theta>0 .
$$

Then $\mathcal{Q}\left(\mathbf{M}_{ \pm}(W, \Psi ; \theta)\right)=0($ for any $\theta>0)$.

Proof of Theorem 6.1. Let $f_{V^{( \pm)}}, h_{V^{( \pm)}}$, and $c_{7}\left(V^{( \pm)}\right)$be as in Lemmas 4.2 and 4.3. We denote

$$
c_{7}^{\prime}=\max \left\{c_{7}\left(V^{(+)}\right), c_{7}\left(V^{(-)}\right)\right\}, \quad c_{8}^{\prime}=\frac{1}{6} c_{1}^{2}\left(c_{1}+4\left(c_{7}^{\prime}\right)^{2}\right)^{-1}
$$

(then $\left.c_{8}^{\prime} \in\left(0, \frac{1}{6} c_{1}\right]\right)$. Suppose $\mu \geq \mu_{0}>0$, where $\mu_{0}$ is a sufficiently large number to be chosen later. To start with, we assume that $\mu_{0} \geq 4 \pi$. Let $\varphi_{ \pm} \in \widetilde{H}^{1}(K)$, and let $k \in \mathbb{R}^{2}$. For $a \in[2 \pi, \mu / 2]$, we denote

$$
\varphi_{ \pm}^{(a)}=\widehat{P}^{T^{ \pm}(a)} \varphi_{ \pm}, \quad \widetilde{\varphi}_{ \pm}^{(a)}=\widehat{P}^{\mathbb{Z}^{2} \backslash T^{ \pm}(a)} \varphi_{ \pm},
$$


where $T^{ \pm}(a)=\left\{N \in \mathbb{Z}^{2}: G_{N}^{ \pm}(k ; \mu) \leq a\right\}$ (the functions $G_{N}^{ \pm}(k ; \mu)$ and $G_{N}(k ; \mu), N \in \mathbb{Z}^{2}$, and also the norms $\|\cdot\|_{*}$ and $\|\cdot\|_{*, \pm}$, were defined at the beginning of $\S 4$ ). Lemma 4.1 yields the estimates

$$
\begin{gathered}
c_{1}\left\|\varphi_{ \pm}^{(a)}\right\|_{*}^{2}=c_{1}\left\|\varphi_{ \pm}^{(a)}\right\|_{*, \pm}^{2} \leq\left\|\left(\widehat{d}_{ \pm}(k)+i \mu \mathcal{H}\right) \varphi_{ \pm}^{(a)}\right\|^{2} \leq c_{2}\left\|\varphi_{ \pm}^{(a)}\right\|_{*, \pm}^{2}=c_{2}\left\|\varphi_{ \pm}^{(a)}\right\|_{*}^{2}, \\
c_{1}\left\|\widetilde{\varphi}_{ \pm}^{(a)}\right\|_{*, \pm}^{2} \leq\left\|\left(\widehat{d}_{ \pm}(k)+i \mu \mathcal{H}\right) \widetilde{\varphi}_{ \pm}^{(a)}\right\|^{2} \leq c_{2}\left\|\widetilde{\varphi}_{ \pm}^{(a)}\right\|_{*, \pm}^{2} .
\end{gathered}
$$

We choose a number $a_{0}^{\prime} \geq 2 \pi$ for which

$$
\max \left\{h_{V^{(+)}}^{2}\left(a_{0}^{\prime}\right), h_{V^{(-)}}^{2}\left(a_{0}^{\prime}\right)\right\} \leq \frac{1}{6} c_{8}^{\prime}
$$

(this can be done because $h_{V^{( \pm)}}(t) \rightarrow 0$ as $t \rightarrow+\infty$ ). Let $a_{1} \geq a_{0}^{\prime}$, and let $\delta=$ $\min \left\{\frac{1}{32} c_{1}, \frac{3}{2} c_{8}^{\prime}\right\}$. We denote by $J$ the smallest integer with $c_{2}^{2} \leq J \delta^{2}$ and choose numbers $a_{2}, \ldots, a_{J+1}$ so that $a_{j+1}>a_{j}(j=1, \ldots, J)$ and each of the (four) functions $\mathcal{P}=$ $\mathcal{G}^{2}+\mathcal{F}^{2}, \mathcal{P}=(\mathcal{G} \pm i \mathcal{F}) \mathcal{H}$, and $\mathcal{P}=\mathcal{H}^{2}$ in the space $L^{\infty}(K) \subset L^{2}(K)$ satisfies the inequalities

$$
a_{j}\left(\sum_{N \in \mathbb{Z}^{2}: 2 \pi|N|>a_{j+1}-a_{j}}\left|\mathcal{P}_{N}\right|^{2}\right)^{1 / 2} \leq \frac{\delta}{4 \sqrt{6 \pi}},
$$

where the $\mathcal{P}_{N}$ are the Fourier coefficients of $\mathcal{P}$ (the numbers $a_{0}^{\prime}, \delta$, and $J$ depend on $p$, $q, F, V^{(+)}$and $V^{(-)}$, and the numbers $a_{2}, \ldots, a_{J+1}$ depend on $a_{1}$ and the functions $\mathcal{F}$, $\mathcal{G}, \mathcal{H}, V^{(+)}$, and $\left.V^{(-)}\right)$. Now we specify the choice of $\mu_{0}$. We assume that $\mu_{0} \geq 2 a_{J+1}$,

$$
\max \left\{h_{V^{(+)}}^{2}\left(\mu_{0}\right), h_{V^{(-)}}^{2}\left(\mu_{0}\right)\right\} \leq \frac{1}{54} c_{8}^{\prime},
$$

and, for all $\mu \geq \mu_{0}$ (and all $k \in \mathbb{R}^{2}$,

$$
\mu^{-2} \max \left\{f_{V^{(-)}}^{2}\left(\# T^{+}(\mu / 2)\right), f_{V^{(+)}}^{2}\left(\# T^{-}(\mu / 2)\right)\right\} \leq \frac{1}{24} c_{8}^{\prime} .
$$

By Lemma 4.2 and the estimate $\# T^{ \pm}(\mu / 2)<\frac{3 \pi}{2} \mu^{2}$ (see (4.1) with $a=\mu / 2 \geq \mu_{0} / 2 \geq 2 \pi$ ), condition (6.6) can indeed be ensured if $\mu_{0}$ is chosen sufficiently large. Since

$$
\sum_{j=1}^{J}\left\|\widetilde{\varphi}_{ \pm}^{\left(a_{j}\right)}-\widetilde{\varphi}_{ \pm}^{\left(a_{j+1}\right)}\right\|_{*, \pm}^{2}=\left\|\widetilde{\varphi}_{ \pm}^{\left(a_{1}\right)}-\widetilde{\varphi}_{ \pm}^{\left(a_{J+1}\right)}\right\|_{*, \pm}^{2}=\left\|\widetilde{\varphi}_{ \pm}^{\left(a_{1}\right)}-\widetilde{\varphi}_{ \pm}^{\left(a_{J+1}\right)}\right\|_{*}^{2} \leq\left\|\varphi_{ \pm}\right\|_{*}^{2},
$$

it follows that, depending on the functions $\varphi_{ \pm} \in \widetilde{H}^{1}(K)$, we can find indices $j_{ \pm} \in$ $\{1, \ldots, J\}$ such that

$$
\left\|\widetilde{\varphi}_{ \pm}^{\left(a_{j_{ \pm}}\right)}-\widetilde{\varphi}_{ \pm}^{\left(a_{j_{ \pm}+1}\right)}\right\|_{*, \pm}^{2} \leq J^{-1}\left\|\varphi_{ \pm}\right\|_{*}^{2} .
$$

Then, by Lemma 4.1 and (6.2), we have

$$
\begin{aligned}
& \left\|\left(\widehat{d}_{ \pm}(k)+i \mu \mathcal{H}\right)\left(\widetilde{\varphi}_{ \pm}^{\left(a_{j_{ \pm}}\right)}-\widetilde{\varphi}_{ \pm}^{\left(a_{j_{ \pm}+1}\right)}\right)\right\| \\
& \quad \leq \sqrt{c_{2}}\left\|\widetilde{\varphi}_{ \pm}^{\left(a_{j_{ \pm}}\right)}-\widetilde{\varphi}_{ \pm}^{\left(a_{j_{ \pm}+1}\right)}\right\|_{*, \pm} \leq\left(\frac{c_{2}}{J}\right)^{1 / 2}\left\|\varphi_{ \pm}\right\|_{*} .
\end{aligned}
$$

The above choice of the indices $j_{ \pm}$is important for the proof of the next lemma.

Lemma 6.2. We have

$$
\left|\left(\left(\widehat{d}_{ \pm}(k)+i \mu \mathcal{H}\right) \widetilde{\varphi}_{ \pm}^{\left(a_{j_{ \pm}}\right)},\left(\widehat{d}_{ \pm}(k)+i \mu \mathcal{H}\right) \varphi_{ \pm}^{\left(a_{j_{ \pm}}\right)}\right)\right| \leq 2 \delta\left\|\varphi_{ \pm}\right\|_{*, \pm}\left\|\varphi_{ \pm}^{\left(a_{j_{ \pm}}\right)}\right\|_{*} .
$$


Proof. From (6.7) we deduce that

$$
\begin{aligned}
\mid\left(\left(\widehat{d}_{ \pm}(k)\right.\right. & \left.+i \mu \mathcal{H}) \widetilde{\varphi}_{ \pm}^{\left(a_{j_{ \pm}}\right)},\left(\widehat{d}_{ \pm}(k)+i \mu \mathcal{H}\right) \varphi_{ \pm}^{\left(a_{j_{ \pm}}\right)}\right) \mid \\
\leq & \left|\left(\left(\widehat{d}_{ \pm}(k)+i \mu \mathcal{H}\right) \widetilde{\varphi}_{ \pm}^{\left(a_{j_{ \pm}+1}\right)},\left(\widehat{d}_{ \pm}(k)+i \mu \mathcal{H}\right) \varphi_{ \pm}^{\left(a_{j_{ \pm}}\right)}\right)\right| \\
& +\left|\left(\left(\widehat{d}_{ \pm}(k)+i \mu \mathcal{H}\right)\left(\widetilde{\varphi}_{ \pm}^{\left(a_{j_{ \pm}}\right)}-\widetilde{\varphi}_{ \pm}^{\left(a_{j_{ \pm}+1}\right)}\right),\left(\widehat{d}_{ \pm}(k)+i \mu \mathcal{H}\right) \varphi_{ \pm}^{\left(a_{j_{ \pm}}\right)}\right)\right| \\
\leq & \left|\left(\left(\widehat{d}_{ \pm}(k)+i \mu \mathcal{H}\right) \widetilde{\varphi}_{ \pm}^{\left(a_{j_{ \pm}+1}\right)},\left(\widehat{d}_{ \pm}(k)+i \mu \mathcal{H}\right) \varphi_{ \pm}^{\left(a_{j_{ \pm}}\right)}\right)\right| \\
& +\left(\frac{c_{2}}{J}\right)^{1 / 2}\left\|\varphi_{ \pm}\right\|\left\|_{*}\right\|\left(\widehat{d}_{ \pm}(k)+i \mu \mathcal{H}\right) \varphi_{ \pm}^{\left(a_{j_{ \pm}}\right)} \| .
\end{aligned}
$$

Also, we have $\left\|\varphi_{ \pm}\right\|_{*} \leq\left\|\varphi_{ \pm}\right\|_{*, \pm}$ and

$$
\left\|\left(\widehat{d}_{ \pm}(k)+i \mu \mathcal{H}\right) \varphi_{ \pm}^{\left(a_{j_{ \pm}}\right)}\right\| \leq \sqrt{c_{2}}\left\|\varphi_{ \pm}^{\left(a_{j_{ \pm}}\right)}\right\|_{*, \pm}=\sqrt{c_{2}}\left\|\varphi_{ \pm}^{\left(a_{j_{ \pm}}\right)}\right\|_{*}
$$

by (6.1). Since

$$
\widehat{d}_{ \pm}(k)+i \mu \mathcal{H}=(\mathcal{G} \pm i \mathcal{F})\left(k_{1}-i \frac{\partial}{\partial x_{1}}\right)+i \mathcal{H}\left(\mu \pm\left(k_{2}-i \frac{\partial}{\partial x_{2}}\right)\right),
$$

we can write (see Lemma 4.5 and inequalities (6.4))

$$
\begin{aligned}
\mid\left(\left(\widehat{d}_{ \pm}(k)+\right.\right. & \left.i \mu \mathcal{H}) \widetilde{\varphi}_{ \pm}^{\left(a_{j_{ \pm}+1}\right)},\left(\widehat{d}_{ \pm}(k)+i \mu \mathcal{H}\right) \varphi_{ \pm}^{\left(a_{j}\right)}\right) \mid \\
\leq & \sqrt{6 \pi} a_{j_{ \pm}}\left(\sum_{N \in \mathbb{Z}^{2}: 2 \pi|N|>a_{j_{ \pm}+1}-a_{j_{ \pm}}}\left|\left(\mathcal{G}^{2}+\mathcal{F}^{2}\right)_{N}\right|^{2}\right)^{1 / 2} \\
& \times\left\|\left(k_{1}-i \frac{\partial}{\partial x_{1}}\right) \widetilde{\varphi}_{ \pm}^{\left(a_{j_{ \pm}+1}\right)}\right\| \cdot\left\|\left(k_{1}-i \frac{\partial}{\partial x_{1}}\right) \varphi_{ \pm}^{\left(a_{j_{ \pm}}\right)}\right\| \\
+ & \sqrt{6 \pi} a_{j_{ \pm}}\left(\sum_{N \in \mathbb{Z}^{2}: 2 \pi|N|>a_{j_{ \pm}+1}-a_{j_{ \pm}}}\left|((\mathcal{G} \mp i \mathcal{F}) \mathcal{H})_{N}\right|^{2}\right)^{1 / 2} \\
& \times\left\|\left(k_{1}-i \frac{\partial}{\partial x_{1}}\right) \widetilde{\varphi}_{ \pm}^{\left(a_{j_{ \pm}+1}\right)}\right\| \cdot\left\|\left(\mu \pm\left(k_{2}-i \frac{\partial}{\partial x_{2}}\right)\right) \varphi_{ \pm}^{\left(a_{j_{ \pm}}\right)}\right\| \\
+ & \sqrt{6 \pi} a_{j_{ \pm}}\left(\sum_{N \in \mathbb{Z}^{2}: 2 \pi|N|>a_{j_{ \pm}+1}-a_{j_{ \pm}}}\left|((\mathcal{G} \pm i \mathcal{F}) \mathcal{H})_{N}\right|^{2}\right)^{1 / 2} \\
& \times\left\|\left(\mu \pm\left(k_{2}-i \frac{\partial}{\partial x_{2}}\right)\right) \widetilde{\varphi}_{ \pm}^{\left(a_{j_{ \pm}+1}\right)}\right\| \cdot\left\|\left(k_{1}-i \frac{\partial}{\partial x_{1}}\right) \varphi_{ \pm}^{\left(a_{j_{ \pm}}\right)}\right\| \\
+ & \sqrt{6 \pi} a_{j_{ \pm}}\left(\sum_{N \in \mathbb{Z}^{2}: 2 \pi|N|>a_{j_{ \pm}+1}-a_{j_{ \pm}}}\left|\left(\mathcal{H}^{2}\right)_{N}\right|^{2}\right)^{1 / 2} \\
& \times\left\|\left(\mu \pm\left(k_{2}-i \frac{\partial}{\partial x_{2}}\right)\right) \widetilde{\varphi}_{ \pm}^{\left(a_{j_{ \pm}+1}\right)}\right\| \cdot\left\|\left(\mu \pm\left(k_{2}-i \frac{\partial}{\partial x_{2}}\right)\right) \varphi_{ \pm}^{\left(a_{j_{ \pm}}\right)}\right\| \\
\leq & \delta\left\|\widetilde{\varphi}_{ \pm}^{\left(a_{j_{ \pm}+1}\right)}\right\|_{*, \pm}\left\|\varphi_{ \pm}^{\left(a_{j_{ \pm}}\right)}\right\|_{*, \pm} \leq \delta\left\|_{\varphi_{ \pm}}\right\|\left\|_{*, \pm}\right\| \varphi_{ \pm}^{\left(a_{j_{ \pm}}\right)} \|_{*} .
\end{aligned}
$$

Now, estimate (6.8) for all $\mu \geq \mu_{0}$ and all $k \in \mathbb{R}^{2}$ follows from (6.9) and the choice of $J \in \mathbb{N}$. Lemma 6.2 is proved.

Now we define a set $\mathbf{M} \subset \mathbb{N}$ (for which $\mathcal{Q}(\mathbb{N} \backslash \mathbf{M})=0$ ). We have $\#\left\{N \in \mathbb{Z}^{2}: \pi|N|<\right.$ $\left.a_{J}\right\} \leq \tau^{*} \doteq 4 \pi^{-1} a_{J}^{2}$. Put $\theta=\frac{1}{3} 2^{-6} c_{1}\left(\pi a_{1}^{2} \tau^{*}\right)^{-1}$. The corollary to Lemma 6.1 implies that for all functions $\mathcal{P}_{ \pm}^{\prime}=(\mathcal{G} \pm i \mathcal{F}) V^{( \pm)} e^{2 \pi i(N, x)}$ and $\mathcal{P}_{ \pm}^{\prime}=\mathcal{H} V^{( \pm)} e^{2 \pi i(N, x)}$, where $N \in \mathbb{Z}^{2}$ and $\pi|N|<a_{J}$, we have $\mathcal{Q}\left(\mathbf{M}_{ \pm}\left(\mathcal{P}_{ \pm}^{\prime}, \Psi ; \theta\right)\right)=0$. Let $\mathbf{M}^{\prime}$ be the union of the sets $\mathbf{M}_{ \pm}\left(\mathcal{P}_{ \pm}^{\prime}, \Psi ; \theta\right)$ for both signs + and - and for all functions $\mathcal{P}_{ \pm}^{\prime}$ as above. Since there are 
at most $4 \tau^{*}$ such sets, we have $\mathcal{Q}\left(\mathbf{M}^{\prime}\right)=0$. We put $\mathbf{M}=\mathbb{N} \backslash\left(\mathbf{M}^{\prime} \cup\left\{m \in \mathbb{N}: \pi m<\mu_{0}\right\}\right)$. Then $\mathcal{Q}(\mathbb{N} \backslash \mathbf{M})=0$, and $\mu \geq \mu_{0}$ whenever $\mu \in \pi \mathbf{M}$. In what follows, we assume that $\mu \in \pi \mathbf{M}$.

Lemma 6.3. For all $\mu \in \pi \mathbf{M}$ (and all $k \in \mathbb{R}^{2}$ ) we have

$$
\left|\left(\left(\widehat{d}_{ \pm}(k)+i \mu \mathcal{H}\right) \varphi_{ \pm}^{\left(a_{j_{ \pm}}\right)}, e^{\mp 2 i \mu \Psi} V^{(\mp)} \varphi_{\mp}^{\left(a_{1}\right)}\right)\right| \leq \frac{c_{1}}{16}\left\|\varphi_{ \pm}^{\left(a_{j_{ \pm}}\right)}\right\|_{*}\left\|\varphi_{\mp}^{\left(a_{1}\right)}\right\| .
$$

Proof. If $\mu \in \pi \mathbf{M}$, then $\mu / \pi \in \mathbb{N} \backslash \mathbf{M}_{ \pm}\left(\mathcal{P}_{ \pm}^{\prime}, \Psi ; \theta\right)$ for all functions $\mathcal{P}_{ \pm}^{\prime}$ indicated above. Therefore, the definition of the set $\mathbf{M}_{ \pm}\left(\mathcal{P}_{ \pm}^{\prime}, \Psi ; \theta\right)$ implies the inequalities

$$
\begin{aligned}
& 6 \pi a_{1}^{2} \sum_{N \in \mathbb{Z}^{2}: \pi|N|<a_{J}}\left|\int_{K} e^{\mp 2 i \mu\left(\Psi-x_{2}\right)}(\mathcal{G} \mp i \mathcal{F}) V^{(\mp)} e^{2 \pi i(N, x)} d^{2} x\right|<6 \pi a_{1}^{2} \tau^{*} \theta=\frac{c_{1}}{32}, \\
& 6 \pi a_{1}^{2} \sum_{N \in \mathbb{Z}^{2}: \pi|N|<a_{J}}\left|\int_{K} e^{\mp 2 i \mu\left(\Psi-x_{2}\right)} \mathcal{H} V^{(\mp)} e^{2 \pi i(N, x)} d^{2} x\right|<6 \pi a_{1}^{2} \tau^{*} \theta=\frac{c_{1}}{32} .
\end{aligned}
$$

Since $\# T^{ \pm}\left(a_{1}\right) \leq 6 \pi a_{1}^{2}$, we deduce the estimate

$$
\begin{aligned}
& \left|\left(\left(\widehat{d}_{ \pm}(k)+i \mu \mathcal{H}\right) \varphi_{ \pm}^{\left(a_{j_{ \pm}}\right)}, e^{\mp 2 i \mu \Psi} V^{(\mp)} \varphi_{\mp}^{\left(a_{1}\right)}\right)\right| \\
& \leq\left|\left((\mathcal{G} \pm i \mathcal{F})\left(k_{1}-i \frac{\partial}{\partial x_{1}}\right) \varphi_{ \pm}^{\left(a_{j_{ \pm}}\right)}, e^{\mp 2 i \mu \Psi} V^{(\mp)} \varphi_{\mp}^{\left(a_{1}\right)}\right)\right| \\
& \quad+\left|\left(\mathcal{H}\left(\mu \pm\left(k_{2}-i \frac{\partial}{\partial x_{2}}\right)\right) \varphi_{ \pm}^{\left(a_{j_{ \pm}}\right)}, e^{\mp 2 i \mu \Psi} V^{(\mp)} \varphi_{\mp}^{\left(a_{1}\right)}\right)\right| \\
& \leq \mid \sum_{N \in T^{ \pm}\left(a_{j_{ \pm}}\right),}\left(k_{1}+2 \pi N_{1}\right) \overline{\left(\varphi_{ \pm}\right)_{N}\left(\varphi_{\mp}\right)_{M}} \\
& \quad \times \int_{K \in T^{\mp}\left(a_{1}\right)} e^{\mp 2 i \mu \Psi+2 \pi i(M-N, x)}(\mathcal{G} \mp i \mathcal{F}) V^{(\mp)} d^{2} x \mid \\
& \quad+\mid \sum_{N \in T^{ \pm}\left(a_{j_{ \pm}}\right),}\left(\mu \pm\left(k_{2}+2 \pi N_{2}\right)\right) \overline{\left(\varphi_{ \pm}\right)_{N}}\left(\varphi_{\mp}\right)_{M} \\
& \quad \times \int_{K \in T^{\mp}\left(a_{1}\right)} e^{\mp 2 i \mu \Psi+2 \pi i(M-N, x)} \mathcal{H} V^{(\mp)} d^{2} x \mid \\
& \leq 6 \pi a_{1}^{2}\left\|\varphi_{ \pm}^{\left(a_{j_{ \pm}}\right)}\right\|\left\|_{*}\right\| \varphi_{\mp}^{\left(a_{1}\right)} \| \\
& \quad \times\left(\sum_{N^{\prime} \in \mathbb{Z}^{2}: \pi\left|N^{\prime}\right|<a_{J}}\left|\int_{K} e^{\mp 2 i \mu\left(\Psi-x_{2}\right)}(\mathcal{G} \mp i \mathcal{F}) V^{(\mp)} e^{2 \pi i\left(N^{\prime}, x\right)} d^{2} x\right|\right. \\
& \left.\quad \sum_{N^{\prime} \in \mathbb{Z}^{2}: \pi\left|N^{\prime}\right|<a_{J}}\left|\int_{K} e^{\mp 2 i \mu\left(\Psi-x_{2}\right)} \mathcal{H} V^{(\mp)} e^{2 \pi i\left(N^{\prime}, x\right)} d^{2} x\right|\right) \\
& \leq \frac{c_{1}}{16}\left\|\varphi_{ \pm}^{\left(a_{j_{ \pm}}\right)}\right\|_{*}\left\|\varphi_{\mp}^{\left(a_{1}\right)}\right\| .
\end{aligned}
$$

This proves Lemma 6.3.

We pass to the proof of the main estimates. In the sequel it is assumed that $k \in \mathbb{R}^{2}$ is such that $k_{1}=\pi$. Taking into account the inequalities

$$
\left\|\varphi_{\mp}^{\left(a_{1}\right)}\right\| \leq\left\|\varphi_{\mp}^{\left(a_{j_{\mp}}\right)}\right\| \leq \pi^{-1}\left\|\varphi_{\mp}^{\left(a_{j_{\mp}}\right)}\right\|_{*} \leq\left\|\varphi_{\mp}^{\left(a_{j_{\mp}}\right)}\right\|_{*},
$$


and using (6.8) and (6.10), we obtain

$$
\begin{aligned}
\|\left(\widehat{d}_{ \pm}\right. & (k)+i \mu \mathcal{H}) \varphi_{ \pm}+e^{\mp 2 i \mu \Psi} V^{(\mp)} \varphi_{\mp}^{\left(a_{1}\right)} \|^{2} \\
= & \left\|\left(\widehat{d}_{ \pm}(k)+i \mu \mathcal{H}\right) \varphi_{ \pm}^{\left(a_{j_{ \pm}}\right)}+\left(\widehat{d}_{ \pm}(k)+i \mu \mathcal{H}\right) \widetilde{\varphi}_{ \pm}^{\left(a_{j_{ \pm}}\right)}+e^{\mp 2 i \mu \Psi} V^{(\mp)} \varphi_{\mp}^{\left(a_{1}\right)}\right\|^{2} \\
\geq & \left\|\left(\widehat{d}_{ \pm}(k)+i \mu \mathcal{H}\right) \varphi_{ \pm}^{\left(a_{j_{ \pm}}\right)}\right\|^{2}+\left\|\left(\widehat{d}_{ \pm}(k)+i \mu \mathcal{H}\right) \widetilde{\varphi}_{ \pm}^{\left(a_{j_{ \pm}}\right)}+e^{\mp 2 i \mu \Psi} V^{(\mp)} \varphi_{\mp}^{\left(a_{1}\right)}\right\|^{2} \\
& -2\left|\left(\left(\widehat{d}_{ \pm}(k)+i \mu \mathcal{H}\right) \widetilde{\varphi}_{ \pm}^{\left(a_{j_{ \pm}}\right)},\left(\widehat{d}_{ \pm}(k)+i \mu \mathcal{H}\right) \varphi_{ \pm}^{\left(a_{j_{ \pm}}\right)}\right)\right| \\
& -2\left|\left(\left(\widehat{d}_{ \pm}(k)+i \mu \mathcal{H}\right) \varphi_{ \pm}^{\left(a_{j_{ \pm}}\right)}, e^{\mp 2 i \mu \Psi} V^{(\mp)} \varphi_{\mp}^{\left(a_{1}\right)}\right)\right| \\
\geq & c_{1}\left\|\varphi_{ \pm}^{\left(a_{j_{ \pm}}\right)}\right\|_{*}^{2}+\left\|\left(\widehat{d}_{ \pm}(k)+i \mu \mathcal{H}\right) \widetilde{\varphi}_{ \pm}^{\left(a_{j_{ \pm}}\right)}+e^{\mp 2 i \mu \Psi} V^{(\mp)} \varphi_{\mp}^{\left(a_{1}\right)}\right\|^{2} \\
& -4 \delta\left\|\varphi_{ \pm}\right\|_{*, \pm}\left\|\varphi_{ \pm}^{\left(a_{j_{ \pm}}\right)}\right\|_{*}-\frac{c_{1}}{8}\left\|\varphi_{ \pm}^{\left(a_{j_{ \pm}}\right)}\right\|_{*}\left\|\varphi_{\mp}^{\left(a_{1}\right)}\right\| \\
\geq & c_{1}\left\|\varphi_{ \pm}^{\left(a_{j_{ \pm}}\right)}\right\|_{*}^{2}+\left\|\left(\widehat{d}_{ \pm}(k)+i \mu \mathcal{H}\right) \widetilde{\varphi}_{ \pm}^{\left(a_{j_{ \pm}}\right)}+e^{\mp 2 i \mu \Psi} V^{(\mp)} \varphi_{\mp}^{\left(a_{1}\right)}\right\|^{2} \\
& -2 \delta\left(\left\|\widetilde{\varphi}_{ \pm}^{\left(a_{j_{ \pm}}\right)}\right\|_{*, \pm}^{2}+2\left\|\varphi_{ \pm}^{\left(a_{j_{ \pm}}\right)}\right\|_{*, \pm}^{2}\right)-\frac{c_{1}}{16}\left(\left\|\varphi_{ \pm}^{\left(a_{j_{ \pm}}\right)}\right\|_{*}^{2}+\left\|\varphi_{\mp}^{\left(a_{j_{\mp}}\right)}\right\|_{*}^{2}\right) .
\end{aligned}
$$

For any $\varepsilon \in(0,1)$, we have (see Lemma 4.3 )

$$
\begin{aligned}
& \left\|\left(\widehat{d}_{ \pm}(k)+i \mu \mathcal{H}\right) \widetilde{\varphi}_{ \pm}^{\left(a_{j_{ \pm}}\right)}+e^{\mp 2 i \mu \Psi} V^{(\mp)} \varphi_{\mp}^{\left(a_{1}\right)}\right\|^{2} \\
& \quad \geq(1-\varepsilon)\left\|\left(\widehat{d}_{ \pm}(k)+i \mu \mathcal{H}\right) \widetilde{\varphi}_{ \pm}^{\left(a_{j_{ \pm}}\right)}\right\|^{2}-(1-\varepsilon) \varepsilon^{-1}\left\|V^{(\mp)} \varphi_{\mp}^{\left(a_{1}\right)}\right\|^{2} \\
& \quad \geq(1-\varepsilon) c_{1}\left\|\widetilde{\varphi}_{ \pm}^{\left(a_{j_{ \pm}}\right)}\right\|_{*, \pm}^{2}-(1-\varepsilon) \varepsilon^{-1}\left(c_{7}\left(V^{(\mp)}\right)\right)^{2}\left\|\varphi_{\mp}^{\left(a_{1}\right)}\right\|_{*}^{2} \\
& \quad \geq(1-\varepsilon) c_{1}\left\|\widetilde{\varphi}_{ \pm}^{\left(a_{j_{ \pm}}\right)}\right\|_{*, \pm}^{2}-(1-\varepsilon) \varepsilon^{-1}\left(c_{7}^{\prime}\right)^{2}\left\|\varphi_{\mp}^{\left(a_{j_{\mp}}\right)}\right\|_{*}^{2} .
\end{aligned}
$$

Therefore,

$$
\begin{aligned}
& \sum_{+,-}\left\|\left(\widehat{d}_{ \pm}(k)+i \mu \mathcal{H}\right) \varphi_{ \pm}+e^{\mp 2 i \mu \Psi} V^{(\mp)} \varphi_{\mp}^{\left(a_{1}\right)}\right\|^{2} \\
& \geq\left(c_{1}-(1-\varepsilon) \varepsilon^{-1}\left(c_{7}^{\prime}\right)^{2}-4 \delta-\frac{c_{1}}{8}\right) \sum_{+,-}\left\|\varphi_{ \pm}^{\left(a_{j_{ \pm}}\right)}\right\|_{*}^{2} \\
& \quad+\left((1-\varepsilon) c_{1}-2 \delta\right) \sum_{+,-}\left\|\widetilde{\varphi}_{ \pm}^{\left(a_{j_{ \pm}}\right)}\right\|_{*, \pm}^{2} \cdot
\end{aligned}
$$

If $c_{7}^{\prime}>0$, we put $\varepsilon=4\left(c_{7}^{\prime}\right)^{2}\left(c_{1}+4\left(c_{7}^{\prime}\right)^{2}\right)^{-1}$. Since $(1-\varepsilon) \varepsilon^{-1}\left(c_{7}^{\prime}\right)^{2}=c_{1} / 4,4 \delta \leq c_{1} / 8$ and $2 \delta \leq \frac{1}{2}(1-\varepsilon) c_{1}=3 c_{8}^{\prime}$, we have

$$
\begin{gathered}
\sum_{+,-}\left\|\left(\widehat{d}_{ \pm}(k)+i \mu \mathcal{H}\right) \varphi_{ \pm}+e^{\mp 2 i \mu \Psi} V^{(\mp)} \varphi_{\mp}^{\left(a_{1}\right)}\right\|^{2} \\
\geq \frac{c_{1}}{2} \sum_{+,-}\left\|\varphi_{ \pm}^{\left(a_{j_{ \pm}}\right)}\right\|_{*}^{2}+3 c_{8}^{\prime} \sum_{+,-}\left\|\widetilde{\varphi}_{ \pm}^{\left(a_{j_{ \pm}}\right)}\right\|_{*, \pm}^{2},
\end{gathered}
$$

which is true even if $c_{7}^{\prime}=0$. Estimate (6.11) is a key point in the proof of Theorem 6.1, and now it is not too hard to complete the proof. For $\varphi_{ \pm} \in \widetilde{H}^{1}(K)$, we have

$$
\widetilde{\varphi}_{ \pm}^{\left(a_{1}\right)}=\widehat{P}^{T^{ \pm}(\mu / 2) \backslash T^{ \pm}\left(a_{1}\right)} \varphi_{ \pm}+\widehat{P}^{\mathbb{Z}^{2} \backslash\left(T^{+}(\mu / 2) \cup T^{-}(\mu / 2)\right)} \varphi_{ \pm}+\widehat{P}^{T^{\mp}(\mu / 2)} \varphi_{ \pm} .
$$


Using Lemmas 4.2, 4.3, and 4.4 and conditions (6.3), (6.5), and (6.6), we obtain

$$
\begin{aligned}
& \left\|V^{(\mp)} \widehat{P}^{T^{\mp}(\mu / 2) \backslash T^{\mp}\left(a_{1}\right)} \varphi_{\mp}\right\|^{2} \leq h_{V^{(\mp)}}^{2}\left(a_{1}\right)\left\|\widehat{P}^{T^{\mp}(\mu / 2) \backslash T^{\mp}\left(a_{1}\right)} \varphi_{\mp}\right\|_{*}^{2} \\
& \quad \leq \frac{1}{6} c_{8}^{\prime}\left\|\widehat{P}^{T^{\mp}(\mu / 2) \backslash T^{\mp}\left(a_{1}\right)} \varphi_{\mp}\right\|_{*}^{2}, \\
& \left\|V^{(\mp)} \widehat{P}^{\mathbb{Z}^{2} \backslash\left(T^{+}(\mu / 2) \cup T^{-}(\mu / 2)\right)} \varphi_{\mp}\right\|^{2} \leq 9 h_{V^{(\mp)}}^{2}(\mu)\left\|\widehat{P}^{\mathbb{Z}^{2} \backslash\left(T^{+}(\mu / 2) \cup T^{-}(\mu / 2)\right)} \varphi_{\mp}\right\|_{*}^{2} \\
& \quad \leq \frac{1}{6} c_{8}^{\prime}\left\|\widehat{P}^{\mathbb{Z}^{2} \backslash\left(T^{+}(\mu / 2) \cup T^{-}(\mu / 2)\right)} \varphi_{\mp}\right\|_{*}^{2}, \\
& \left\|V^{(\mp)} \widehat{P}^{T^{ \pm}(\mu / 2)} \varphi_{\mp}\right\|^{2} \leq f_{V(\mp)}^{2}\left(\# T^{ \pm}(\mu / 2)\right)\left\|\widehat{P}^{T^{ \pm}(\mu / 2)} \varphi_{\mp}\right\|^{2} \\
& \quad \leq 4 \mu^{-2} f_{V^{(\mp)}}^{2}\left(\# T^{ \pm}(\mu / 2)\right)\left\|\widehat{P}^{T^{ \pm}(\mu / 2)} \varphi_{\mp}\right\|_{*, \mp}^{2} \leq \frac{1}{6} c_{8}^{\prime}\left\|\widehat{P}^{T^{ \pm}(\mu / 2)} \varphi_{\mp}\right\|_{*, \mp}^{2} .
\end{aligned}
$$

Therefore,

$$
\begin{aligned}
\sum_{+,-} & \left\|\left(\widehat{d}_{ \pm}(k)+i \mu \mathcal{H}\right) \varphi_{ \pm}+e^{\mp 2 i \mu \Psi} V^{(\mp)} \varphi_{\mp}\right\|^{2} \\
\geq & \frac{1}{2} \sum_{+,-}\left\|\left(\widehat{d}_{ \pm}(k)+i \mu \mathcal{H}\right) \varphi_{ \pm}+e^{\mp 2 i \mu \Psi} V^{(\mp)} \varphi_{\mp}^{\left(a_{1}\right)}\right\|^{2} \\
& -3 \sum_{+,-}\left\|V^{(\mp)} \widehat{P}^{T^{\mp}(\mu / 2) \backslash T^{\mp}\left(a_{1}\right)} \varphi_{\mp}\right\|^{2} \\
& -3 \sum_{+,-}\left\|V^{(\mp)} \widehat{P}^{\mathbb{Z}^{2} \backslash\left(T^{+}(\mu / 2) \cup T^{-}(\mu / 2)\right)} \varphi_{\mp}\right\|^{2} \\
& -3 \sum_{+,-}\left\|V^{(\mp)} \widehat{P}^{T^{ \pm}(\mu / 2)} \varphi_{\mp}\right\|^{2} \\
\geq & \frac{1}{2} \sum_{+,-}\left\|\left(\widehat{d}_{ \pm}(k)+i \mu \mathcal{H}\right) \varphi_{ \pm}+e^{\mp 2 i \mu \Psi} V^{(\mp)} \varphi_{\mp}^{\left(a_{1}\right)}\right\|^{2} \\
& -\frac{1}{2} c_{8}^{\prime} \sum_{+,-}\left\|\widetilde{\varphi}_{\mp}^{\left(a_{1}\right)}\right\|_{*, \mp}^{2} .
\end{aligned}
$$

Finally, from $(6.11),(6.12)$, and the condition $c_{8}^{\prime} \leq \frac{1}{6} c_{1}$ we deduce the estimate claimed in Theorem 6.1:

$$
\begin{aligned}
& \sum_{+,-}\left\|\left(\widehat{d}_{ \pm}(k)+i \mu \mathcal{H}\right) \varphi_{ \pm}+e^{\mp 2 i \mu \Psi} V^{(\mp)} \varphi_{\mp}\right\|^{2} \\
& \quad \geq \frac{c_{1}}{4} \sum_{+,-}\left\|\varphi_{ \pm}^{\left(a_{j_{ \pm}}\right)}\right\|_{*, \pm}^{2}+\frac{3}{2} c_{8}^{\prime} \sum_{+,-}\left\|\widetilde{\varphi}_{ \pm}^{\left(a_{j_{ \pm}}\right)}\right\|_{*, \pm}^{2}-\frac{1}{2} c_{8}^{\prime} \sum_{+,-}\left\|\widetilde{\varphi}_{\mp}^{\left(a_{1}\right)}\right\|_{*, \mp}^{2} \\
& \quad \geq \frac{c_{1}}{6} \sum_{+,-}\left\|\varphi_{ \pm}^{\left(a_{j_{ \pm}}\right)}\right\|_{*, \pm}^{2}+c_{8}^{\prime} \sum_{+,-}\left\|\widetilde{\varphi}_{ \pm}^{\left(a_{j_{ \pm}}\right)}\right\|_{*, \pm}^{2}+\frac{1}{2} c_{8}^{\prime} \sum_{+,-}\left\|\varphi_{ \pm}^{\left(a_{1}\right)}\right\|_{*, \pm}^{2} \\
& \geq \frac{c_{1}}{6} \sum_{+,-}\left\|\varphi_{ \pm}^{\left(a_{j_{ \pm}}\right)}\right\|_{*}^{2}+c_{8}^{\prime} \sum_{+,-}\left\|\widetilde{\varphi}_{ \pm}^{\left(a_{j_{ \pm}}\right)}\right\|_{*, \pm}^{2} \\
& \geq \frac{c_{1}}{6} \sum_{+,-}\left\|\varphi_{ \pm}^{\left(a_{1}\right)}\right\|_{*}^{2}+c_{8}^{\prime} \sum_{+,-}\left\|\widetilde{\varphi}_{ \pm}^{\left(a_{1}\right)}\right\|_{*, \pm}^{2} .
\end{aligned}
$$

Theorem 6.1 is proved. 


\section{REFERENCES}

[1] P. Kuchment, Floquet theory for partial differential equations, Oper. Theory Adv. Appl., vol. 60, Birkhäuser Verlag, Basel, 1993. MR1232660 (94h:35002)

[2] P. Kuchment and S. Levendorskiı̌, On the structure of spectra of periodic elliptic operators, Trans. Amer. Math. Soc. 354 (2002), no. 2, 537-569. MR1862558 (2002g:35163)

[3] L. I. Danilov, On the spectrum of the Dirac operator with periodic potential, Preprint, Fiz.-Tekhn. Inst. Ural. Otdel. Akad. Nauk SSSR, Sverdlovsk, 1987. (Russian)

[4] - A property of the integer lattice in $\mathbf{R}^{3}$ and the spectrum of the Dirac operator with periodic potential, Preprint, Fiz.-Tekhn. Inst. Ural. Otdel. Akad. Nauk SSSR, Sverdlovsk, 1988. (Russian)

[5] - On the spectrum of the Dirac operator in $\mathbf{R}^{n}$ with periodic potential, Teoret. Mat. Fiz. 85 (1990), no. 1, 41-53; English transl., Theoret. and Math. Phys. 85 (1990), no. 1, 1039-1048 (1991). MR 1083951 (92a:35119)

[6] The spectrum of the Dirac operator with periodic potential. I, Fiz.-Tekhn. Inst. Ural. Otdel. Akad. Nauk SSSR, Izhevsk, 1991. (Manuscript dep. VINITI 12.12.91, no. 4588-B91.) (Russian)

[7] The spectrum of the Dirac operator with periodic potential. III, Fiz.-Tekhn. Inst. Ural. Otdel. Ross. Akad. Nauk, Izhevsk, 1992. (Manuscript dep. VINITI 10.07.92, no. 2252-B92.) (Russian)

[8] _ The spectrum of the Dirac operator with periodic potential. VI, Fiz.-Tekhn. Inst. Ural. Otdel. Ross. Akad. Nauk, Izhevsk, 1996. (Manuscript dep. VINITI 31.12.96, no. 3855-B96.) (Russian)

[9] - Resolvent estimates and the spectrum of the Dirac operator with a periodic potential, Teoret. Mat. Fiz. 103 (1995), no. 1, 3-22; English transl., Theoret. and Math. Phys. 103 (1995), no. 1, 349-365. MR1470934 (98f:35112)

[10] _ Absolute continuity of the spectrum of a periodic Dirac operator, Differentsial'nye Uravneniya 36 (2000), no. 2, 233-240; English transl., Differential Equations 36 (2000), no. 2, 262-271. MR:1773794 (2001f:47082)

[11] On the spectrum of the two-dimensional periodic Dirac operator, Teoret. Mat. Fiz. 118 (1999), no. 1, 3-14; English transl., Theoret. and Math. Phys. 118 (1999), no. 1, 1-11. MR1702856 (2000h:35117)

[12] M. Sh. Birman and T. A. Suslina, The periodic Dirac operator is absolutely continuous, Integral Equations and Operator Theory 34 (1999), 377-395. MR1702229 (2000h:47068)

[13] L. I. Danilov, On the spectrum of the periodic Dirac operator, Teoret. Mat. Fiz. 124 (2000), no. 1, 317; English transl., Theoret. and Math. Phys. 124 (2000), no. 1, 859-871. MR.1821309 (2002b:81028)

[14] M. Sh. Birman and T. A. Suslina, Two-dimensional periodic magnetic Hamiltonian is absolutely continuous, Algebra i Analiz 9 (1997), no. 1, 32-48; English transl., St. Petersburg Math. J. 9 (1998), no. 1, 21-32. MR1458417 (98g:47038)

[15] _ Absolute continuity of the two-dimensional periodic magnetic Hamiltonian with discontinuous vector-valued potential, Algebra i Analiz 10 (1998), no. 4, 1-36; English transl., St. Petersburg Math. J. 10 (1999), no. 4, 579-601. MR1654063(99k:81060)

[16] I. S. Lapin, Absolute continuity of the spectra of two-dimensional periodic magnetic Schrödinger operator and Dirac operator with potentials in the Zygmund class, Probl. Mat. Anal., vyp. 22, S.Peterburg. Univ., St. Petersburg, 2001, pp. 74-105; English transl., J. Math. Sci. (New York) 106 (2001), no. 3, 2952-2974. MR1906028 (2003h:35182)

[17] A. V. Sobolev, Absolute continuity of the periodic magnetic Schrödinger operator, Invent. Math. 137 (1999), 85-112. MR1703339 (2000g:35028)

[18] M. Sh. Birman and T. A. Suslina, Periodic magnetic Hamiltonian with variable metric. The problem of absolute continuity, Algebra i Analiz 11 (1999), no. 2, 1-40; English transl., St. Petersburg Math. J. 11 (2000), no. 2, 203-232. MR1702587 (2000i:35026)

[19] L. I. Danilov, On the absolute continuity of the spectrum of periodic Schrödinger and Dirac operators. I, Fiz.-Tekhn. Inst. Ural. Otdel. Ross. Akad. Nauk, Izhevsk, 2000. (Manuscript dep. VINITI 15.06.00, no. 1683-B00.) (Russian)

[20] _ On the absolute continuity of the spectrum of a periodic Schrödinger operator, Mat. Zametki 73 (2003), no. 1, 49-62; English transl., Math. Notes 73 (2003), no. 1-2, 46-57. MR.1993539 (2004f:35130)

[21] Z. Shen, On absolute continuity of the periodic Schrödinger operators, Internat. Math. Res. Notices 2001, no. 1, 1-31. MR1809495(2002a:47078)

[22] _ Absolute continuity of periodic Schrödinger operators with potentials in the Kato class, Illinois J. Math. 45 (2001), no. 3, 873-893. MR1879241 (2002m:25036)

[23] The periodic Schrödinger operators with potentials in the Morrey class, J. Funct. Anal. 193 (2002), no. 2, 314-345. MR.1929505 (2003k:47071) 
[24] L. Friedlander, On the spectrum of a class of second order periodic elliptic differential operators, Comm. Math. Phys. 229 (2002), 49-55. MR1917673 (2003k:35179)

[25] T. A. Suslina and R. G. Shterenberg, Absolute continuity of the spectrum of the Schrödinger operator with the potential concentrated on a periodic system of hypersurfaces, Algebra i Analiz 13 (2001), no. 5, 197-240; English transl., St. Petersburg Math. J. 13 (2002), no. 5, 859-891. MR1882869 (2002m:35172)

[26] H. L. Cycon, R. G. Froese, W. Kirsch, and B. Simon, Schrödinger operators with application to quantum mechanics and global geometry, Springer-Verlag, Berlin 1987. MR0883643 (88g:35003)

[27] A. Morame, Absence of singular spectrum for a perturbation of a two-dimensional Laplace-Beltrami operator with periodic electro-magnetic potential, J. Phys. A 31 (1998), 7593-7601. MR1652918 (99i:81039)

[28] L. I. Danilov, On absolute continuity of the spectrum of periodic Schrödinger and Dirac operators. II, Fiz.-Tekhn. Inst. Ural. Otdel. Ross. Akad. Nauk, Izhevsk, 2001. (Manuscript dep. VINITI 09.04.01, no. 916-B2001.) (Russian)

[29] - On the spectrum of the two-dimensional periodic Schrödinger and Dirac operators, Izv. Inst. Mat. i Inform. Udmurt. Univ., vyp. 3 (26), Izhevsk, 2002, pp. 3-98. (Russian)

[30] _ On the spectrum of the two-dimensional periodic Schrödinger operator, Teoret. Mat. Fiz. 134 (2003), no. 3, 447-459. (Russian) MR2001818 (2004j:35210)

[31] _ On absolute continuity of the spectrum of periodic Schrödinger and Dirac operators. III, Fiz.-Tekhn. Inst. Ural. Otdel. Ross. Akad. Nauk, Izhevsk, 2002. (Manuscript dep. VINITI 22.10.02, no. 1798-B2002.) (Russian)

[32] _ On the absence of eigenvalues in the spectrum of two-dimensional periodic Dirac and Schrödinger operators, Izv. Inst. Mat. i Inform. Udmurt. Univ., vyp. 1 (29), Izhevsk, 2004, pp. 4984. (Russian)

[33] M. Sh. Birman, T. A. Suslina, and R. G. Shterenberg, Absolute continuity of the spectrum of a two-dimensional Schrödinger operator with potential supported on a periodic system of curves, Algebra i Analiz 12 (2000), no. 6, 140-177; English transl., St. Petersburg Math. J. 12 (2001), no. 6, 983-1012. MR1816514(2002k:35227)

[34] R. G. Shterenberg, Absolute continuity of a two-dimensional magnetic periodic Schrödinger operator with electric potential of measure derivative type, Zap. Nauchn. Sem. S.-Peterburg. Otdel. Mat. Inst. Steklov. (POMI) 271 (2000), 276-312; English transl., J. Math. Sci. (New York) 115 (2003), no. 6, 2862-2882. MR.1810620 (2002m:35171)

[35] _ - Absolute continuity of the spectrum of two-dimensional periodic Schrödinger operators with positive electric potential, Algebra i Analiz 13 (2001), no. 4, 196-228; English transl., St. Petersburg Math. J. 13 (2002), no. 4, 659-683. MR1865502 (2002j:35230)

[36] _ Absolute continuity of the spectrum of a two-dimensional magnetic periodic Schrödinger operator with positive electric potential, Trudy S.-Peterburg. Mat. Obshch. 9 (2001), 199-233; English transl. in Amer. Math. Soc. Transl. Ser. 2, vol. 209, Amer. Math. Soc., Providence, RI, 2003.

[37] _ Absolute continuity of spectra of two-dimensional periodic Schrödinger operators with strongly subordinate magnetic potentials, Report no. 21, 2002/2003, Mittag-Leffler Inst., Stockholm, 2002.

[38] T. A. Suslina and R. G. Shterenberg, Absolute continuity of the spectrum of the magnetic Schrödinger operator with metric in a two-dimensional periodic waveguide, Algebra i Analiz 14 (2002), no. 2, 159-206; English transl., St. Petersburg Math. J. 14 (2003), no. 2, 305-343. MR1925885 (2003h:35185)

[39] R. G. Shterenberg, Schrödinger operator in a periodic waveguide on the plane and quasi-conformal mappings, Zap. Nauchn. Sem. S.-Peterburg. Otdel. Mat. Inst. Steklov. (POMI) 295 (2003), 204-243. (Russian)

[40] A. V. Sobolev and J. Walthoe, Absolute continuity in periodic waveguides, Proc. London Math. Soc. (3) 85 (2002), no. 3, 717-741. MR1936818 (2003j:35240)

[41] E. Shargorodsky and A. V. Sobolev, Quasi-conformal mappings and periodic spectral problems in dimension two, LANL Archives: math.SP/0109216 (2001).

[42] L. Thomas, Time dependent approach to scattering from impurities in a crystal, Comm. Math. Phys. 33 (1973), 335-343. MR0334766 (48:13084)

[43] I. M. Gel'fand, Expansion in characteristic functions of an equation with periodic coefficients, Dokl. Akad. Nauk SSSR 73 (1950), no. 6, 1117-1120. (Russian) MR0039154 (12:503a) 
[44] M. Reed and B. Simon, Methods of modern mathematical physics. IV. Analysis of operators, Acad. Press, New York-London, 1978. MR0493421 (58:12429c)

[45] _ Methods of modern mathematical physics. III. Scattering theory, Acad. Press, New YorkLondon, 1979. MR0529429 (90m:81085)

Physical-Technical Institute, Ural Branch of the Russian Academy of Sciences, Kirov Street 132, Izhevsk 426000, Russia

E-mail address: danilov@otf.pti.udm.ru

Received 12/JAN/2004

Translated by A. PLOTKIN 